\title{
A One-Pot Selective Saturation and Functionalization of Heteroaro- matics Leading to Dihydropyridines and Dihydroquinolines
}

\author{
Dong Wang, ${ }^{,}{ }^{\dagger}$ Yuanyang Jiang, ${ }^{\dagger}$ Linru Dong, Gaoyu Li, ${ }^{\dagger}$ Baoying Sun, Laurent Désaubry ${ }^{\dagger, \ddagger}$ and Peng \\ $\mathrm{Yu}^{\dagger}$
}

${ }^{\dagger}$ College of Biotechnology, Tianjin University of Science and Technology, Tianjin 300457, China

${ }^{\ddagger}$ Laboratory of Medicinal Chemistry and Cardio-oncology, FRE2033, CNRS, Institut Le Bel, Strasbourg, France

Supporting Information Placeholder

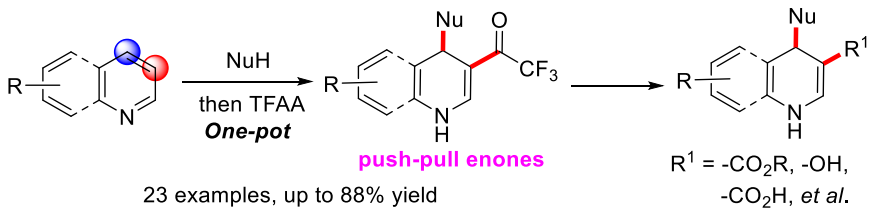

ABSTRACT: A one-pot regioselective two C-C bonds forming dearomatization of pyridines and quinolines is disclosed. Two 3,4betaines are identified for the first time as very useful organic synthons in heterocyclic chemistry. Furthermore, the chemical reactivity of the prepared trifluoromethyl ketones, a new type of push-pull enones, has been explored to develop straightforward methods for their functionalization. This protocol represents a breakthrough in the dearomatization of heteroaromatics as both the selective saturation and functionalization of heteroaromatics are achieved in high efficiency by the attachment of two substituents, including the valuable trifluoromethyl ketone group.

\section{INTRODUCTION}

A recent review published in Science on synthetic chemistry in the pharmaceutical industry stated that "Key unsolved problems in synthetic chemistry included selective saturation and functionalization of heteroaromatics...". Here, we tackled both of these issues by developing an unprecedented regioselective C-alkylation of pyridines and quinolines coupled to the introduction of a ketone moiety to provide highly functionalized dihydropyridines (DHPs) and dihydroquinolines (DHQs).
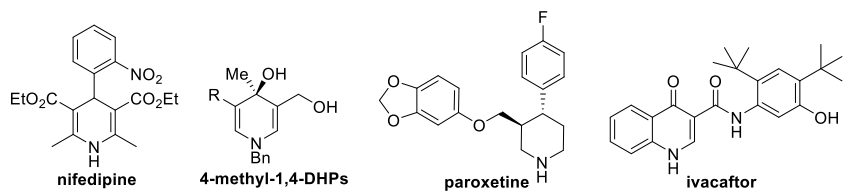

Figure 1. Selected 3,4-Disubstituted DHPs, DHQs and Piperidines

Both 1,4-DHPs and 1,4-DHQs are highly sought-after structural motifs because they are widely used as building blocks in synthetic chemistry (mainly converted to piperidines) or as pharmacophores in medicinal chemistry (Figure 1). The 1,4-DHPs, as one of the most important classes of calcium channel modulators, are widely used in treating cardiovascular diseases. ${ }^{2}$ Furthermore, they also represent synthetic mimics of the natural nicotinamide cofactors. ${ }^{3}$ For example, the chiral 4-methyl-1,4-DHPs shown in Figure 1 are identified as efficient NADH mimics by reduction of benzoylformic ester. ${ }^{4}$ The 1,4-DHQs exhibit wide range of biological activities. 5

Due to the ready availability of starting materials, simple synthetic manipulations and the step economy in comparison to annulative chemistry, the reductive dearomatization of the corresponding pyridines or quinolines continues to be prominent. A wellestablished method to access 1,4-DHPs is through the sodium dithionite reduction of pre-functionalized pyridinium salts (Scheme 1A). ${ }^{6}$ Despite these advantages, only $\mathrm{C}-\mathrm{H}$ bonds are formed in this approach, leading to minimum structure complexity. Besides, this method is restricted to electron deficient pyridines.

Scheme 1. Dearomatization Approach to DHPs and DHQs.
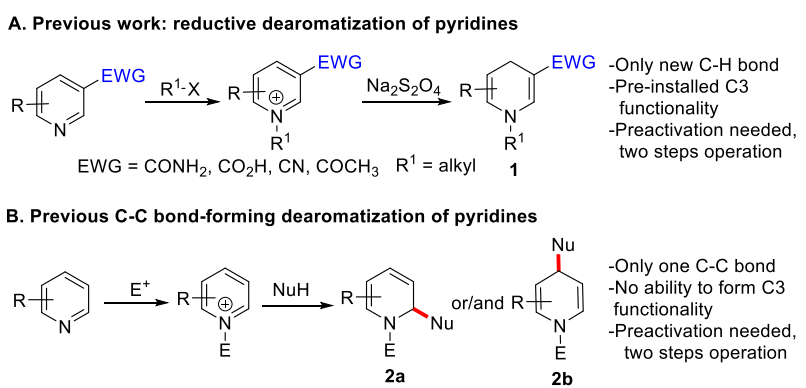

C. Bennasar and Bosch: $2 \times \mathrm{C}-\mathrm{C}$ bonds-forming dearomatization of $\mathrm{N}$-alkyl pyridinium salts

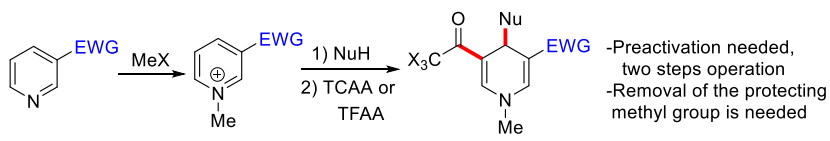

D. This work: 2 X C-C bonds-forming dearomatization of pyridines and quinolines

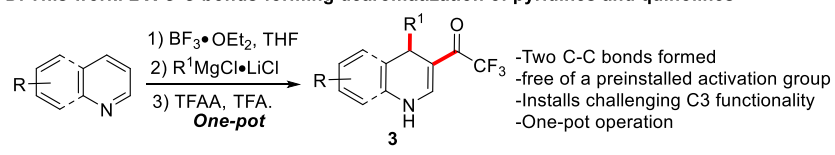

In comparison with the reductive dearomatization, most $\mathrm{C}-\mathrm{C}$ bond-forming pyridine dearomatization is performed by nucleophilic addition to the activated quaternary pyridinium salts generated through stoichiometric functionalization of the pyridine nitrogen with strong electrophiles (Scheme 1B). ${ }^{7}$ Despite this protocol is more efficient than the reductive dearomatization, it only forms one $\mathrm{C}-\mathrm{C}$ bond with the more electrophilic $\mathrm{C} 2$ or $\mathrm{C} 4$ carbon of 
pyridines, precluding the ability to prepare C3 substituted azaheterocycles. The C3 substituted aza-heterocycles, however, are demanding targets as the most $S_{N} A r$ only allow the functionalization of the electrophilic $\mathrm{C} 2$ and $\mathrm{C} 4$ positions of the arene. ${ }^{8}$ Notably, Bennasar and Bosch reported a one-pot synthesis of C3substitued DHPs by C4-selective addition of lithium enolates or aryl cuprates to $\mathrm{N}$-alkyl pyridinium salts followed by TFAA or TCAA quench (Scheme 1C). ${ }^{9}$ However, the removal of the methyl group on nitrogen is needed in a late stage and can be difficult in many cases. Therefore, the development of a general $\mathrm{C}-\mathrm{C}$ bondforming dearomatization enabling $\mathrm{C} 3$-functionalization free of a preinstalled activation group in one-pot operation would represent a significant synthetic advancement.

Thanks to the pioneering studies by Buchwald ${ }^{10}$ and You, ${ }^{11} \mathrm{C}-\mathrm{C}$ bond-forming dearomatization without preactivation of the heterocycle has emerged as an attractive alternative for the traditional dearomatization. Inspired by the impressive advances, and as a continuation of our research on the efficient synthesis of drug-like nitrogen heterocycles, ${ }^{12}$ we describe herein a regioselective difunctionalization of pyridines and quinolines, providing convenient access to DHPs and DHQs substituted by a trifluoromethyl ketone under mild conditions and in one pot (Scheme 1D). Besides, the prepared DHPs and DHQs are trifluoromethyl ketones, which would have great potential in both synthetic and medicinal chemistry. $^{13}$

For the first time, we have identified two 3,4-betaines as very useful organic synthons, whose corresponding equivalent reagents are pyridines or quinolines (Figure 2). Furthermore, the efficiency of this one pot dearomative protocol is remarkable. Two C-C bonds, one $\mathrm{C}=\mathrm{C}$ bond and one $\mathrm{N}-\mathrm{H}$ bond are formed; one $\mathrm{C}=\mathrm{C}$ bond and one $\mathrm{C}=\mathrm{N}$ bond are broken simultaneously. To the best of our knowledge, no precedent exists for the preparation of DHPs or DHQs via a one-pot two C-C bonds forming dearomatization.
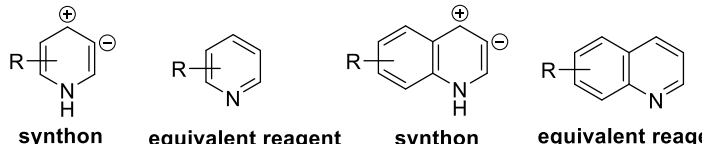

equivalent reage

synthon

Figure 2. 3,4-Betaine Synthons in Heterocyclic Chemistry

\section{RESULTS AND DISCUSSION}

Initially, quinoline was subjected to the 1,4-dearomatization conditions to generate the enamine intermediate, ${ }^{14}$ which would react with TFAA and $\mathrm{Et}_{3} \mathrm{~N}$ to afford the amide product 3a' (Table 1). After careful characterization of the formed product, we were surprised to find out that instead of 3a', the C3-acylation product 3a was actually formed (Table 1 , entry 1 ). Considering the imperative need for $\mathrm{C} 3$ functionalization of DHPs or DHQs and the valuable potential of trifluoromethyl ketones in both synthetic and medicinal chemistry, ${ }^{13}$ we decided to optimize this reaction further.

Table 1. Reaction Optimization of the C-C Bond Forming Dearomatization*

\begin{tabular}{cccc} 
entry & additive/eq & eq (TFAA) & yield, $\%$ \\
1 & $\mathrm{Et}_{3} \mathrm{~N} / 3$ & 3 & 50 \\
2 & $\mathrm{TFA} / 3$ & 3 & 83 \\
3 & $\mathrm{TFA} / 1.5$ & 3 & 61 \\
4 & $\mathrm{TFA} / 3$ & 2 & 81 \\
\hline
\end{tabular}

$\begin{array}{lccc}5 & \text { TFA/3 } & 1.1 & 60 \\ 6 & \text { TFA/3 } & 1.5 & 63 \\ 7 & \text { TFA/4 } & 2 & 85 \\ 8 & \text { TFA/6 } & 2 & 88\end{array}$

* All reactions were conducted with quinoline $(\sim 100 \mathrm{mg}, 1.0$ equiv), $\mathrm{BF}_{3} \cdot \mathrm{OEt}_{2}$ (1.1 equiv), $\mathrm{RMgCl} \cdot \mathrm{LiCl}(1.2$ equiv), THF $(0.5$ $\mathrm{M})$; TFAA and additive.

Replacing $\mathrm{Et}_{3} \mathrm{~N}$ with TFA resulted in a sharp increase in the product yield ( $83 \%$, entry 2 ), and reducing the quantity of TFA was unfavorable for the product formation ( $61 \%$, entry 3$)$, indicating that acidic conditions are critical for this transformation, probably due to the enhanced electrophilicity of TFAA under acidic conditions. Attempts were made to decrease the TFAA amount (entries 4-6), and it was found that 2 equiv is best $(81 \%$, entry 4). Finally, we found that TFA has no significant effect on the product yield with more than 3 equiv loading (entries 7-8). Considering the balance between large excess TFA and product yield, condition in entry 4 was chosen as the optimum one. If the yield is lower than $50 \%$ utilizing 3 equiv of TFA, 6 equiv of TFA would be applied.

Following the establishment of the optimum conditions, the substrate scope of this dearomative transformation was investigated (Scheme 2). A variety of the desired 3,4-disubstituted DHQs are accessible in modest to good yields (up to 88\%) utilizing this reaction. Excellent regioselectivity was also observed (C4 vs. C2). Functional groups such as ester group (3g), alkyl group (3e), alkoxy group (3f), and phenyl group (3i) are tolerated, as are halides (3b-3d, 3l and 3m) that would normally be reduced in the reductive dearomatizations. Substituents at various positions, including 5-, 6-, and 7-, are all accommodated. Furthermore, it seems that those quinolines with neutral electron density $(\mathbf{3 a}, \mathbf{3 b}-\mathbf{3 e}, \mathbf{3 i})$ are the best substrates as very low yields were obtained for quinolines with strong electron donating group (3f) or electron withdrawing group (3g). Interestingly, although little conversion (28\% yield) was observed for $\mathbf{3 g}$ under the optimum conditions, modest yield could be obtained ( $62 \%$ yield) under basic conditions.

Next, this dearomatization was further applied to pyridine derivatives and we are delighted to find out that a variety of 3,4,5trisubstituted DHPs bearing two different electron-withdrawing groups at the $\beta$-positions could be obtained using this strategy (up to $79 \%$ yield, Scheme 2). Moreover, the regioselectivity is excellent as none $\mathrm{C} 2$ or C6 alkylation product could be detected. With regard to the scope of pyridines, an electron withdrawing group attached at $\mathrm{C} 3$ position is required, which would increase the electrophilicity of the $\mathrm{C} 4$ position, leading to better reactivity with Grignard reagents. Moreover, the stronger electron deficiency, the better the reactivity (i.e., $\mathbf{3 l}>\mathbf{3 j}>\mathbf{3 k}$ ). Completely different from quinolines, various Grignard reagents can react with pyridines. Except for alkyl Grignard reagents $(\mathbf{3 j}-\mathbf{3 m})$, both alkenyl (3o) and aryl Grignard reagents (3p-3v) are applicable substrates. Generally speaking, aryl Grignard reagents offer the best yield, followed by alkyl Grignard reagents (i.e., $\mathbf{3 p}>\mathbf{3 j}>\mathbf{3 o}$ ). Furthermore, those strong electron deficient aromatic Grignard reagents don't react (i.e., $\mathbf{3 w}$ ). The low yield for $\mathbf{3 s}$ is probably due to steric reasons. 
Scheme 2. Substrate Scope for C-C Bond Forming Dearomatization of Pyridines and Quinolines.*

$$
\frac{1) \mathrm{BF}_{3} \cdot \mathrm{OEt}_{2}, \mathrm{THF}, 0^{\circ} \mathrm{C}}{2) \mathrm{R}^{1} \mathrm{MgCl} \cdot \mathrm{LiCl},-50^{\circ} \mathrm{C} \text { or }-30^{\circ} \mathrm{C}}
$$<smiles></smiles>

3a: $81 \%, 88 \%^{a}$<smiles>CC(C)C1C(C(=O)C(F)(F)F)=CNc2cccc(Br)c21</smiles>

3b: $55 \%^{\mathrm{a}}, 66 \%$ (BRSM)<smiles>CC(C)C1C(C(=O)C(F)(F)F)=CNc2ccc(Br)cc21</smiles>

3c: $65 \%^{a}, 75 \%$ (BRSM)<smiles>CC(C)C1C(C(=O)C(F)(F)F)=CNc2cc(Br)ccc21</smiles>

3d: $74 \%$ a<smiles>Cc1ccc2c(c1)C(C(C)C)C(C(=O)C(F)(F)F)=CN2</smiles>

3e: $47 \%^{a}, 98 \%$ (BRSM)<smiles>COc1ccc2c(c1)C(C(C)C)C(C(=O)C(F)(F)F)=CN2</smiles>

3f: $23 \%^{a}, 51 \%$ (BRSM)<smiles>CC(=O)c1ccc2c(c1)C(C(C)C)C(C(=O)C(F)(F)F)=CN2</smiles>

3g: $28 \%{ }^{a}, 73 \%$ (BRSM) $62 \%^{b}, 89 \%$ (BRSM)<smiles>CC(C)C1C(Cl)=CNC=C1C(=O)C(F)(F)F</smiles>

3m: $51 \%^{a}$<smiles>O=C(C1=CNc2ccccc2C1C1CCCCC1)C(F)(F)F</smiles>

3h: $11 \%^{a}, 27 \%$ (BRSM)<smiles>CC(C)C1C(C(=O)C(F)(F)F)=CNc2ccc(-c3ccccc3)cc21</smiles>

3i: $66 \%$<smiles>CC(C)C1C(C#N)=CNC=C1C(=O)F</smiles>

3j: $62 \%$<smiles>COC(=O)C1=CNC=C(C(=O)C(F)(F)F)C1C(C)C</smiles>

3k: $32 \%^{a}$<smiles>CCOCC1=C(Cl)NC=C(C(=O)C(F)(F)F)C1C(C)C</smiles>

31: $73 \%$<smiles>CC1=CNC=C(C(=O)OC(F)(F)F)C1c1c(C)cc(C)cc1C</smiles>

3s: $40 \%^{a}, 80 \%$ (BRSM)<smiles>Cc1ccccc1C1C(C(=O)OC(F)(F)F)=CNC=C1C(=O)c1ccccc1</smiles>

3n: $22 \%$<smiles>C=C(C)C1C(C#N)=CNC=C1C(=O)C(F)(F)F</smiles>

3o: $23 \%^{a}, 53 \%$ (BRSM)<smiles>Cc1ccccc1C1C(C#N)=CNC=C1C(=O)C(F)(F)F</smiles>

3p: $78 \%$<smiles>N#CC1=CNC=C(C(=O)C(F)(F)F)C1c1ccccc1</smiles>

3q: $47 \%$<smiles>Cc1ccc(C2C(C#N)=CNC=C2C(=O)C(F)(F)F)cc1</smiles>

3r: $60 \%, 75 \%$ (BRSM)<smiles>COc1cccc(C2C(C#N)=CNC=C2C(=O)C(F)(F)F)c1</smiles><smiles>COc1ccc(C2C(C#N)=CNC=C2C(=O)C(F)(F)F)cc1</smiles><smiles>N#CC1=CNC=C(C(=O)C(F)(F)F)C1c1cccc(F)c1</smiles><smiles>N#CC1=CNC=C(C(=O)C(F)(F)F)C1c1ccc(F)cc1</smiles>

3u: $36 \%, 70 \%$ (BRSM)

3w: $0 \%$

*Unless otherwise noted, all reactions were conducted with quinoline or pyridine derivative ( $250 \mathrm{mg}, 1.0$ equiv $), \mathrm{BF}_{3} \bullet \mathrm{OEt}_{2}(1.1 \mathrm{equiv})$, $\mathrm{RMgCl} \cdot \mathrm{LiCl}$ (1.2 equiv), THF $(0.5 \mathrm{M})$; TFAA (2.0 equiv), TFA (3.0 equiv). BRSM is the yield based on the recovered starting material. ${ }^{a}$ 6.0 equiv of TFA was used. ${ }^{b}$ Instead of TFA, TEA (3.0 equiv) was used.

Scheme 3. Preliminary Study with Other Electrophiles

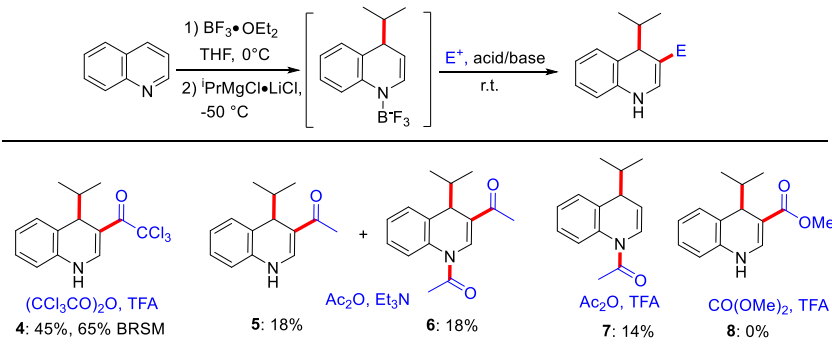

To further demonstrate that this protocol extends beyond addition of TFAA, preliminary experiments with other electrophiles were performed (Scheme 3). Trichloroacetic anhydride reacted and gave medium yield. We were surprised to find out that the amide product 7 was formed in $14 \%$ yield using acetic anhydride under the developed conditions, and several polar by-products were formed. Interestingly, both the expected product 5 and the double acylated product 6 were formed as major products under basic acylation conditions. Dimethyl carbonate did not react in this transformation.

Scheme 4. Derivatization of the Prepared DHQs

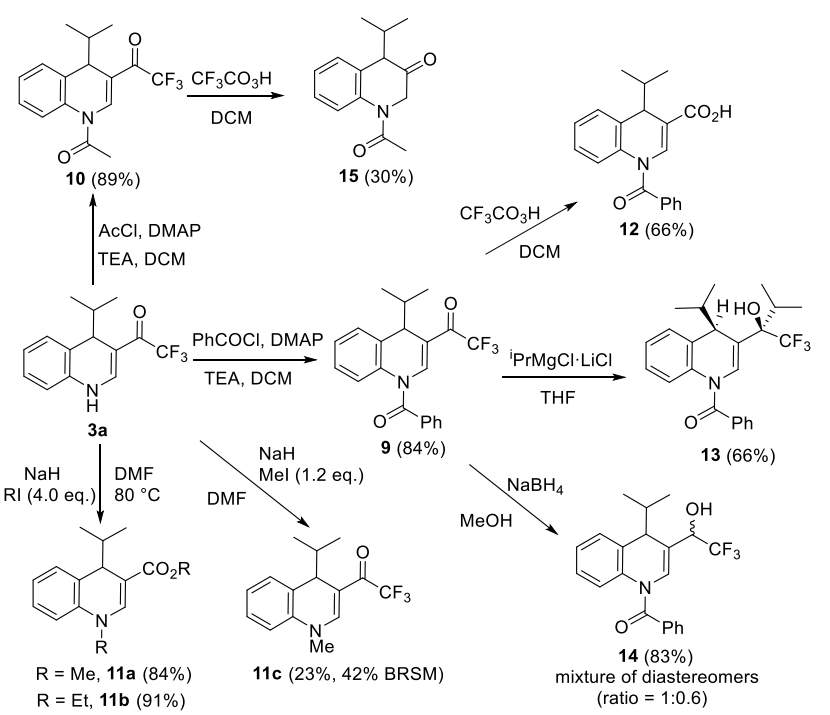

It is worth noting that the prepared 3-trifluoroacetyl substituted DHQs and DHPs are a new type of push-pull enones, whose chemistry is underexplored and would exhibit special reactivity. ${ }^{15}$ Indeed, we were pleased to find out that manipulation of the trifluoroacetyl group generates a diverse set of functionalized DHQs and DHPs. Using 3a as the model compound for DHQs, the trifluoroacetyl group can be easily converted to various common func- 
tional groups, such as ester (11a-b, Scheme 4), alcohol (13 \& 14), ketone (15) and carboxylic acid (12). The trifluoroacetyl group seems to be reluctant to undergo hydrolysis reactions as attempts to convert 3a to methyl ester by treating it with $\mathrm{NaOMe}$ in methanol under reflux failed. Whereas $\mathbf{3 a}$ could be converted to $\mathrm{N}$ alkylated methyl ester (11a) in $84 \%$ yield by a modified procedure $^{16}$ for hydrolyzing the trifluoroacetyl group. Alkylation with EtI also gave good yield (11b, 91\% yield). Moreover, the selective alkylation of the nitrogen atom in $\mathbf{3 a}$ to produce 11c could be realized by running the reaction with 1.2 equiv of $\mathrm{MeI}$ at $\mathrm{rt}$, indicating that the alkylation of nitrogen is preferred.

Compound 3a was protected by benzoyl and acetyl group to obtain 9 and 10 in high yields. As far as we know, the BaeyerVilliger reaction involving $\alpha, \beta$-unsaturated trifluoroacetyl group has never been disclosed. In our case it gave unexpected results. Using the $N$-benzoylated DHP 9 gave the carboxylic acid $\mathbf{1 2}$ in $66 \%$ yield. Surprisingly, with the $N$-acylated DHP 10, the ketone 15, resulting from the migration of the vinyl group, was produced as the major product. It should be pointed out that peroxytrifluoroacetic acid used in these reactions was generated in situ by reaction of urea hydrogen peroxide with TFAA. Finally, the transformation of enones to the corresponding allylic alcohols was investigated. The reaction of non-fluorinated enones with Grignard reagents is reported to lead to regioisomeric mixtures. ${ }^{17}$ Moreover, it is reported that cyclic $\beta$-alkoxy- $\alpha, \beta$-unsaturated trifluoromethyl ketones, as typical push-pull enones, react predominantly with Grignard reagents in 1,4 -addition mode. ${ }^{18}$ Similar selectivity was also observed for trifluoromethylated $\alpha$ bromoenones. ${ }^{19}$ Unexpectingly, the Grignard addition to compound $\mathbf{9}$ predominantly led to the 1,2-addition product $\mathbf{1 3}$ in $66 \%$ yield. In addition, although the preexisting stereocenter is at $\beta$ position of the carbonyl group, the reaction is still highly stereoselective. Next, reduction of the carbonyl group was examined. Reduction of $\mathbf{9}$ with $\mathrm{NaBH}_{4}$ afforded $\mathbf{1 4}$ as a diastereomeric mixture in $83 \%$ yield without the need of any Lewis acid, which is different from the conventional Luche reduction requiring $\mathrm{CeCl}_{3}$ to catalyze the selective 1,2-reduction of enones. ${ }^{20}$

Scheme 5. Derivatization of the Prepared DHPs

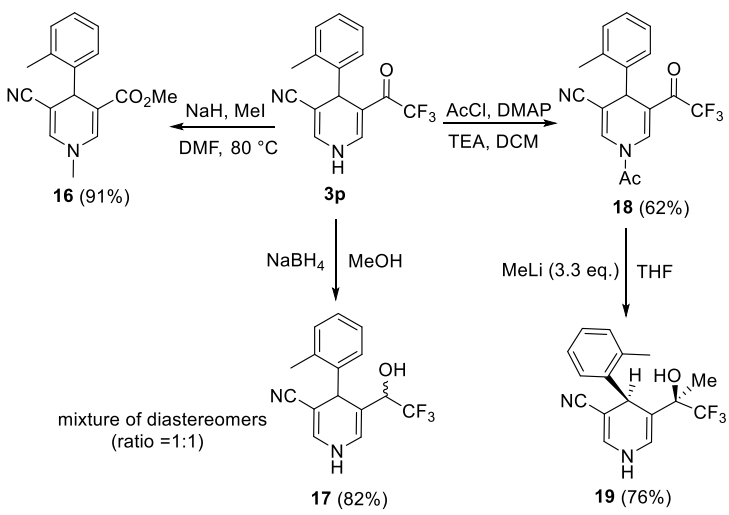

Similar transformations with the prepared DHPs, as exemplified by $\mathbf{3 p}$, were also successful (Scheme 5). Hydrolysis or reduction of $\mathbf{3 p}$ afforded $\mathbf{1 6}$ or $\mathbf{1 7}$ in high yields. Reaction of $\mathbf{1 8}$ with excess methyl lithium produced the $N$-deacylated tertiary alcohol 19 in $76 \%$ yield with high stereoselectivity.

\section{CONCLUSION}

In conclusion, we have developed a one-pot regioselective two $\mathrm{C}-\mathrm{C}$ bonds forming dearomatization of pyridines and quinolines, providing convenient access to 3,4-disubstituted DHPs and DHQs. The trifluoroacetyl group of DHPs and DHQs was found to be easily converted to various functional groups. This method not only provides the concise and efficient synthesis of functionalized
DHPs and DHQs, but also offers a new strategy for the selective saturation and functionalization of nitrogen heteroaromatics.

\section{EXPERIMENTAL SECTION}

General Remarks. The preparation experiments were performed under an argon atmosphere in oven dried glassware. Solvents used as reaction media were distilled immediately before use: THF were distilled from $\mathrm{Na} /$ benzophenone ketyl, DCM and DCE was distilled from calcium hydride, DMF were obtained from vacuum distillation. All reagents were purchased at the highest commercial quality and used without further purification. Oil bath was used as the heat source for those reactions that require heating. Reactions were monitored by thin layer chromatography (TLC) using ultra violet light (UV) as the visualizing agent. Nuclear magnetic resonance spectra (NMR) were recorded on Bruker AV-400 instruments and were calibrated using residual undeuterated solvent as an internal reference $\left({ }^{1} \mathrm{H}\right.$ NMR: $\mathrm{CHC}_{3} 7.26 \mathrm{ppm}$, ${ }^{13} \mathrm{C}$ NMR: $\left.\mathrm{CHCl}_{3} 77.16 \mathrm{ppm}\right)$. High resolution mass spectra (HRMS) were recorded on a hybrid IT-TOF mass spectrometer (Shimadzu LCMS-IT-TOF, Kyoto, Japan). The following abbreviations were used to indicate multiplicities: $\mathrm{s}=$ singlet, $\mathrm{d}=\mathrm{dou}-$ blet, $\mathrm{t}=$ triplet, $\mathrm{q}=$ quartet, quin = quintet, sex = sextet, sep = septet, $\mathrm{dd}=$ doublet of doublets, $\mathrm{dt}=$ doublet of triplets, ddd = doublet of doublet of doublets, $\mathrm{ddt}=$ doublet of doublet of triplets, $\mathrm{m}=$ multiplet).

General procedure for the synthesis of 3,4-disubstituted dihydropyridines and dihydroquinolines using alkyl Grignard reagents (GP1). A dry and argon flushed $25 \mathrm{ml}$ flask, equipped with a magnetic stirring bar and a rubber septum is charged with a solution of a quinoline or pyridine derivative (1.0 eq.) in dry THF $(0.5 \mathrm{M})$ and cooled to $0{ }^{\circ} \mathrm{C} . \mathrm{BF}_{3} \cdot \mathrm{OEt}_{2}(1.1 \mathrm{eq}$. $)$ is added dropwise and stirred for $15 \mathrm{~min}$ at the same temperature. The reaction mixture is cooled to $-50{ }^{\circ} \mathrm{C}$ followed by dropwise addition of a THF solution of an alkyl Grignard reagent (1.2 eq.), and stirring the reaction mixture at the same temperature for $30 \mathrm{~min}$. Then TFAA ( 2 equiv) and TFA ( 3 or 6 equiv) are added, and the reaction mixture is increased to r.t. by removing the EA/dry ice bath. Stirring is continued at r.t. until the reaction is complete as indicated by TLC. Finally, it is quenched with saturated ammonia water solution (the same volume as methanol) and extracted with EA three times. The combined organic phases are washed with brine, dried over $\mathrm{Na}_{2} \mathrm{SO}_{4}$, and concentrated in vacuo to give the crude product. Purification by flash chromatography furnishes the desired product 3 .

General procedure for the synthesis of 3,4-disubstituted dihydropyridines using alkenyl or aryl Grignard reagents (GP2). A dry and argon flushed $25 \mathrm{ml}$ flask, equipped with a magnetic stirring bar and a rubber septum is charged with a solution of a quinoline or pyridine derivative $(1.0$ eq.) in dry THF $(0.5 \mathrm{M})$ and cooled to $0{ }^{\circ} \mathrm{C} . \mathrm{BF}_{3} \cdot \mathrm{OEt}_{2}$ (1.1 eq.) is added dropwise and stirred for $15 \mathrm{~min}$ at the same temperature. The reaction mixture is cooled to $-30{ }^{\circ} \mathrm{C}$ followed by dropwise addition of an alkenyl or aryl Grignard reagent (1.2 eq.), and stirring the reaction mixture at the same temperature for $2 \mathrm{~h}$. Then TFAA ( 2 equiv) and TFA ( 3 or 6 equiv) are added, and the reaction mixture is increased to r.t. by removing the $\mathrm{CH}_{3} \mathrm{CN} /$ dry ice bath. Stirring is continued at r.t. until the reaction is complete as indicated by TLC. Finally, it is quenched with saturated ammonia water solution (the same volume as methanol) and extracted with EA three times. The combined organic phases are washed with brine, dried over $\mathrm{Na}_{2} \mathrm{SO}_{4}$, and concentrated in vacuo to give the crude product. Purification by flash chromatography furnishes the desired product $\mathbf{3}$.

2,2,2-Trifluoro-1-(4-isopropyl-1,4-dihydroquinolin-3-yl)ethan1-one (3a): Following GP1, using quinoline (250 mg, $1.94 \mathrm{mmol}$ ), $\mathrm{BF}_{3} \cdot \mathrm{OEt}_{2}(302 \mathrm{mg}, 2.13 \mathrm{mmol})$, isopropylmagnesium chloride lithium chloride complex solution $(1.8 \mathrm{ml}, 1.3 \mathrm{M}$ in THF, 2.33 
mmol), TFAA (815 mg, $3.88 \mathrm{mmol}$ ) and TFA (664 mg, 5.82 mmol), the reaction mixture was stirred at r.t. for $2 \mathrm{~h}$. Purification by flash chromatography (PE:EA = 100:1 5:1) furnishes the desired product (423 mg, 81\% yield) as a yellow solid. ${ }^{1} \mathrm{H}$ NMR $\left(400 \mathrm{MHz}, \mathrm{CDCl}_{3}\right) \delta 7.76(\mathrm{~d}, J=6.4 \mathrm{~Hz}, 1 \mathrm{H}), 7.71(\mathrm{~s}, 1 \mathrm{H}), 7.09$ $7.26(\mathrm{~m}, 3 \mathrm{H}), 6.87(\mathrm{~d}, J=7.6 \mathrm{~Hz}, 1 \mathrm{H}), 4.03(\mathrm{~d}, J=3.6 \mathrm{~Hz}, 1 \mathrm{H})$, $1.80-1.86(\mathrm{~m}, 1 \mathrm{H}), 0.88(\mathrm{~d}, J=6.8 \mathrm{~Hz}, 3 \mathrm{H}), 0.67(\mathrm{~d}, J=6.8 \mathrm{~Hz}$, $3 \mathrm{H}) .{ }^{13} \mathrm{C}\{1 \mathrm{H}\} \mathrm{NMR}\left(100 \mathrm{MHz}, \mathrm{CDCl}_{3}\right) \delta 177.1(\mathrm{q}, J=33 \mathrm{~Hz})$, $144.3(\mathrm{q}, J=5 \mathrm{~Hz}), 135.8,130.5,127.4,124.9,123.2,117.9$ (q, $J$ $=290 \mathrm{~Hz}), 115.5,106.7,40.7,35.7,19.7,17.7$. HRMS (-ESI-TOF) $\mathrm{m} / \mathrm{z}$ : [M-H] ${ }^{-}$Calcd for $\mathrm{C}_{14} \mathrm{H}_{13} \mathrm{NOF}_{3}$ 268.0955; Found 268.0953.

(5-Bromo-4-isopropyl-1,4-dihydroquinolin-3-yl)-2,2,2-

trifluoroethan-1-one $(\mathbf{3 b})$ : Following GP1, using 5bromoquinoline $(250 \mathrm{mg}, 1.21 \mathrm{mmol}), \mathrm{BF}_{3} \cdot \mathrm{OEt}_{2}(187 \mathrm{mg}, 1.33$ mmol), isopropylmagnesium chloride lithium chloride complex solution (1.1 ml, 1.3 M in THF, $1.45 \mathrm{mmol}$ ), TFAA (504 mg, 2.42 $\mathrm{mmol})$ and TFA (821 $\mathrm{mg}, 7.26 \mathrm{mmol})$, the reaction mixture was stirred at r.t. overnight. Purification by flash chromatography (PE:EA = 100:1 1:1) furnishes the desired product $(232 \mathrm{mg}, 55 \%$ yield) as a yellow solid, as well as the recovered 5bromoquinoline $(40 \mathrm{mg}$ ), the yield based on the recovered starting material is $66 \%$. ${ }^{1} \mathrm{H}$ NMR (400 MHz, d6-DMSO) $\delta 10.52(\mathrm{~d}, J=$ $6.0 \mathrm{~Hz}, 1 \mathrm{H}), 7.84(\mathrm{~d}, J=6.0 \mathrm{~Hz}, 1 \mathrm{H}), 7.41(\mathrm{~d}, J=7.6 \mathrm{~Hz}, 1 \mathrm{H})$, $7.18(\mathrm{t}, J=7.6 \mathrm{~Hz}, 1 \mathrm{H}), 7.11(\mathrm{~d}, J=7.6 \mathrm{~Hz}, 1 \mathrm{H}), 4.31(\mathrm{~d}, J=4.0$ $\mathrm{Hz}, 1 \mathrm{H}), 1.81-1.86(\mathrm{~m}, 1 \mathrm{H}), 0.77(\mathrm{~d}, J=6.8 \mathrm{~Hz}, 3 \mathrm{H}), 0.60(\mathrm{~d}, J=$ $6.8 \mathrm{~Hz}, 3 \mathrm{H}) .{ }^{13} \mathrm{C}\{1 \mathrm{H}\} \mathrm{NMR}(100 \mathrm{MHz}, \mathrm{d} 6-\mathrm{DMSO}) \delta 175.2$ (q, $J$ $=32 \mathrm{~Hz}), 145.2(\mathrm{q}, J=4 \mathrm{~Hz}), 137.6,128.7,124.0,123.7,117.6(\mathrm{q}$, $J=290 \mathrm{~Hz}), 115.5,101.6,38.8,35.7,20.0,16.5$. HRMS (+ESITOF) $\mathrm{m} / \mathrm{z}$ : $[\mathrm{M}+\mathrm{Na}]^{+}$Calcd for $\mathrm{C}_{14} \mathrm{H}_{13} \mathrm{NOF}_{3} \mathrm{BrNa} 370.0025$; Found 370.0024 .

(6-Bromo-4-isopropyl-1,4-dihydroquinolin-3-yl)-2,2,2-

trifluoroethan-1-one (3c): Following GP1, using 6bromoquinoline $(250 \mathrm{mg}, 1.21 \mathrm{mmol}), \mathrm{BF}_{3} \cdot \mathrm{OEt}_{2}(187 \mathrm{mg}, 1.33$ $\mathrm{mmol}$ ), isopropylmagnesium chloride lithium chloride complex solution (1.1 ml, 1.3 M in THF, $1.45 \mathrm{mmol}$ ), TFAA (504 mg, 2.42 $\mathrm{mmol}$ ) and TFA (821 $\mathrm{mg}, 7.26 \mathrm{mmol})$, the reaction mixture was stirred at r.t. overnight. Purification by flash chromatography (PE:EA = 100:1 1:1) furnishes the desired product $(274 \mathrm{mg}, 65 \%$ yield) as a yellow solid, as well as the recovered 6bromoquinoline $(33 \mathrm{mg})$, the yield based on the recovered starting material is $75 \%$. ${ }^{1} \mathrm{H}$ NMR (400 MHz, d6-DMSO) $\delta 10.39(\mathrm{~d}, J=$ $6.0 \mathrm{~Hz}, 1 \mathrm{H}), 7.80(\mathrm{~d}, J=6.0 \mathrm{~Hz}, 1 \mathrm{H}), 7.39-7.42(\mathrm{~m}, 2 \mathrm{H}), 7.03(\mathrm{~d}$, $J=8.4 \mathrm{~Hz}, 1 \mathrm{H}), 3.92(\mathrm{~d}, J=3.6 \mathrm{~Hz}, 1 \mathrm{H}), 1.66-1.70(\mathrm{~m}, 1 \mathrm{H}), 0.77$ $(\mathrm{d}, J=6.8 \mathrm{~Hz}, 3 \mathrm{H}), 0.59(\mathrm{~d}, J=6.8 \mathrm{~Hz}, 3 \mathrm{H}) .{ }^{13} \mathrm{C}\{1 \mathrm{H}\} \mathrm{NMR}(100$ MHz, d6-DMSO) $\delta 174.7(\mathrm{q}, J=32 \mathrm{~Hz}), 144.6(\mathrm{q}, J=5 \mathrm{~Hz})$, $135.5,132.2,130.0,125.0,117.8,117.5(\mathrm{q}, J=291 \mathrm{~Hz}), 115.8$, 103.7, 35.2, 18.9, 17.5. HRMS (-ESI-TOF) m/z: [M-H] Calcd for $\mathrm{C}_{14} \mathrm{H}_{12} \mathrm{NOF}_{3} \mathrm{Br}$ 346.0060; Found 346.0049.

(7-Bromo-4-isopropyl-1,4-dihydroquinolin-3-yl)-2,2,2trifluoroethan-1-one (3d): Following GP1, using 7bromoquinoline $(250 \mathrm{mg}, 1.21 \mathrm{mmol}), \mathrm{BF}_{3} \cdot \mathrm{OEt}_{2}(187 \mathrm{mg}, 1.33$ mmol), isopropylmagnesium chloride lithium chloride complex solution (1.1 ml, 1.3 M in THF, $1.45 \mathrm{mmol}$ ), TFAA (504 mg, 2.42 mmol) and TFA ( $821 \mathrm{mg}, 7.26 \mathrm{mmol})$, the reaction mixture was stirred at r.t. for $1.5 \mathrm{~h}$. Purification by flash chromatography (PE:EA = 100:1 1:1) furnishes the desired product $(310 \mathrm{mg}, 74 \%$ yield) as a yellow solid. ${ }^{1} \mathrm{H}$ NMR $\left(400 \mathrm{MHz}, \mathrm{CDCl}_{3}\right) \delta 7.67(\mathrm{~d}, J$ $=6.4 \mathrm{~Hz}, 1 \mathrm{H}), 7.21-7.23(\mathrm{~m}, 1 \mathrm{H}), 7.11(\mathrm{~s}, 1 \mathrm{H}), 6.99-7.02(\mathrm{~m}, 2 \mathrm{H})$, $3.97(\mathrm{~d}, J=3.6 \mathrm{~Hz}, 1 \mathrm{H}), 1.77-1.87(\mathrm{~m}, 1 \mathrm{H}), 0.87(\mathrm{~d}, J=6.8 \mathrm{~Hz}$, $3 \mathrm{H}), 0.66(\mathrm{~d}, J=6.8 \mathrm{~Hz}, 3 \mathrm{H}) .{ }^{13} \mathrm{C}\{1 \mathrm{H}\} \mathrm{NMR}\left(100 \mathrm{MHz}, \mathrm{CDCl}_{3}\right)$ $\delta 177.4,143.0(\mathrm{q}, J=5 \mathrm{~Hz}), 137.1,131.9,127.6,122.0,120.5$, 118.0, $117.5(\mathrm{q}, J=290 \mathrm{~Hz}), 107.5,40.3,35.5,19.6,17.6$. HRMS (+ESI-TOF) $m / z$ : $[\mathrm{M}+\mathrm{Na}]^{+}$Calcd for $\mathrm{C}_{14} \mathrm{H}_{13} \mathrm{NOF}_{3} \mathrm{BrNa} 370.0025$; Found 370.0029 .

2,2,2-Trifluoro-1-(4-isopropyl-6-methyl-1,4-dihydroquinolin-3yl)ethan-1-one (3e): Following GP1, using 6-methylquinoline
(300 mg, $2.10 \mathrm{mmol}$ ), $\mathrm{BF}_{3} \cdot \mathrm{OEt}_{2}(326 \mathrm{mg}, 2.30 \mathrm{mmol}$ ), isopropylmagnesium chloride lithium chloride complex solution $(1.93 \mathrm{ml}$, $1.3 \mathrm{M}$ in THF, $2.51 \mathrm{mmol}$ ), TFAA ( $881 \mathrm{mg}, 4.19 \mathrm{mmol}$ ) and TFA $(1.44 \mathrm{~g}, 12.60 \mathrm{mmol})$, the reaction mixture was stirred at r.t. overnight. Purification by flash chromatography (PE:EA = 30:1 20:1) furnishes the desired product ( $279 \mathrm{mg}, 47 \%$ yield) as a yellow solid, as well as the recovered 6-methylquinoline $(157 \mathrm{mg})$, the yield based on the recovered starting material is $98 \% .{ }^{1} \mathrm{H}$ NMR $\left(400 \mathrm{MHz}, \mathrm{CDCl}_{3}\right) \delta 7.68(\mathrm{~d}, J=6.8 \mathrm{~Hz}, 1 \mathrm{H}), 6.99(\mathrm{~d}, J=7.6 \mathrm{~Hz}$, 2H), $6.95(\mathrm{~s}, 1 \mathrm{H}), 6.71(\mathrm{~d}, J=8.0 \mathrm{~Hz}, 1 \mathrm{H}), 3.98(\mathrm{~d}, J=4.0 \mathrm{~Hz}$, $1 \mathrm{H}), 2.33(\mathrm{~s}, 3 \mathrm{H}), 1.78-1.86(\mathrm{~m}, 1 \mathrm{H}), 0.87(\mathrm{~d}, J=7.2 \mathrm{~Hz}, 3 \mathrm{H})$, $0.66(\mathrm{~d}, J=6.8 \mathrm{~Hz}, 3 \mathrm{H}) .{ }^{13} \mathrm{C}\{1 \mathrm{H}\} \operatorname{NMR}\left(100 \mathrm{MHz}, \mathrm{CDCl}_{3}\right) \delta$ $176.8(\mathrm{q}, J=32 \mathrm{~Hz}), 144.9(\mathrm{q}, J=5 \mathrm{~Hz}), 134.7,133.5,130.9$, $127.9,123.3,118.1$ (q, $J=289 \mathrm{~Hz}), 115.6,106.0,40.7,35.8,21.1$, 19.7, 17.7. HRMS (+ESI-TOF) $\mathrm{m} / \mathrm{z}:[\mathrm{M}+\mathrm{Na}]^{+}$Calcd for $\mathrm{C}_{15} \mathrm{H}_{16} \mathrm{NOF}_{3} \mathrm{Na}$ 306.1076; Found 306.1068.

2,2,2-Trifluoro-1-(4-isopropyl-6-methoxy-1,4-dihydroquinolin3-yl)ethan-1-one (3f): Following GP1, using 6-methoxyquinoline (200 mg, $1.26 \mathrm{mmol}$ ), $\mathrm{BF}_{3} \cdot \mathrm{OEt}_{2}$ (196 mg, $1.38 \mathrm{mmol}$ ), isopropylmagnesium chloride lithium chloride complex solution $(1.16 \mathrm{ml}$, $1.3 \mathrm{M}$ in THF, $1.51 \mathrm{mmol})$, TFAA (528 mg, $2.51 \mathrm{mmol})$ and TFA (860 $\mathrm{mg}, 7.54 \mathrm{mmol}$ ), the reaction mixture was stirred at r.t. overnight. Purification by flash chromatography (PE:EA = 50:1 10:1) furnishes the desired product ( $87 \mathrm{mg}, 23 \%$ yield) as a yellow solid, as well as the recovered 6-methoxyquinoline $(111 \mathrm{mg})$, the yield based on the recovered starting material is $51 \%$. ${ }^{1} \mathrm{H}$ NMR $(400$ $\left.\mathrm{MHz}, \mathrm{CDCl}_{3}\right) \delta 8.18(\mathrm{~d}, J=6.0 \mathrm{~Hz}, 1 \mathrm{H}), 7.75(\mathrm{~d}, J=6.8 \mathrm{~Hz}, 1 \mathrm{H})$, $6.75(\mathrm{dd}, \mathrm{J}=2.8,8.8 \mathrm{~Hz}, 1 \mathrm{H}), 6.69(\mathrm{~d}, J=2.4 \mathrm{~Hz}, 1 \mathrm{H}), 4.01(\mathrm{~d}, J$ $=3.6 \mathrm{~Hz}, 1 \mathrm{H}), 3.80(\mathrm{~s}, 3 \mathrm{H}), 1.82-1.89(\mathrm{~m}, 1 \mathrm{H}), 0.90(\mathrm{~d}, J=6.8 \mathrm{~Hz}$, $3 \mathrm{H}), 0.68(\mathrm{~d}, J=6.8 \mathrm{~Hz}, 3 \mathrm{H}) .{ }^{13} \mathrm{C}\{1 \mathrm{H}\}$ NMR $\left(100 \mathrm{MHz}, \mathrm{CDCl}_{3}\right)$ $\delta 176.6(\mathrm{q}, J=32.0 \mathrm{~Hz}), 157.1,144.5(\mathrm{q}, J=5.0 \mathrm{~Hz}), 129.5,124.9$, $118.1(\mathrm{q}, J=290.0 \mathrm{~Hz}), 116.6,115.4,113.0,105.1,55.7,41.0$, 35.9, 20.0, 17.7. HRMS (-ESI-TOF) $\mathrm{m} / \mathrm{z}$ : [M-H] $]^{-}$Calcd for $\mathrm{C}_{15} \mathrm{H}_{15} \mathrm{NO}_{2} \mathrm{~F}_{3}$ 298.1060; Found 298.1047.

Methyl 4-isopropyl-3-(2,2,2-trifluoroacetyl)-1,4dihydroquinoline-6-carboxylate (3g): Following GP1, using quinoline-6-carboxylate (200 mg, $1.07 \mathrm{mmol}), \mathrm{BF}_{3} \cdot \mathrm{OEt}_{2}(167 \mathrm{mg}$, $1.18 \mathrm{mmol}$ ), isopropylmagnesium chloride lithium chloride complex solution (1.48 ml, 1.3 M in THF, $1.92 \mathrm{mmol})$, TFAA (450 $\mathrm{mg}, 2.14 \mathrm{mmol})$ and TFA (730 mg, $6.41 \mathrm{mmol})$, the reaction mixture was stirred at r.t. overnight. Purification by flash chromatography (PE:EA = 100:1 10:1) furnishes the desired product (101 $\mathrm{mg}, 28 \%$ yield) as a yellow solid, as well as the recovered quinoline-6-carboxylate $(121 \mathrm{mg})$, the yield based on the recovered starting material is $73 \% .{ }^{1} \mathrm{H}$ NMR $\left(400 \mathrm{MHz}, \mathrm{CDCl}_{3}\right) \delta 7.89(\mathrm{dd}$, $J=2.0,8.4 \mathrm{~Hz}, 1 \mathrm{H}), 7.84(\mathrm{~s}, 1 \mathrm{H}), 7.67(\mathrm{~d}, \mathrm{~J}=6.4 \mathrm{~Hz}, 1 \mathrm{H}), 6.90(\mathrm{~s}$, $1 \mathrm{H}), 6.83(\mathrm{~d}, \mathrm{~J}=8.4 \mathrm{~Hz}, 1 \mathrm{H}), 4.06(\mathrm{~d}, \mathrm{~J}=4.0 \mathrm{~Hz}, 1 \mathrm{H}), 3.91(\mathrm{~s}$, $3 \mathrm{H}), 1.80-1.88(\mathrm{~m}, 1 \mathrm{H}), 0.88(\mathrm{~d}, J=6.8 \mathrm{~Hz}, 3 \mathrm{H}), 0.67(\mathrm{~d}, J=6.8$ $\mathrm{Hz}, 3 \mathrm{H}) .{ }^{13} \mathrm{C}\{1 \mathrm{H}\} \mathrm{NMR}\left(100 \mathrm{MHz}, \mathrm{CDCl}_{3}\right) \delta 177.3(\mathrm{q}, J=33.0$ $\mathrm{Hz}), 166.7,142.6(\mathrm{q}, J=5.0 \mathrm{~Hz}), 139.7,132.2,129.2,126.4$, 123.0, 117.5 (q, $J=290.0 \mathrm{~Hz}), 114.9,108.1,52.3,40.6,35.7,19.4$, 17.7. HRMS (+ESI-TOF) $m / z: \quad[\mathrm{M}+\mathrm{Na}]^{+}$Calcd for $\mathrm{C}_{16} \mathrm{H}_{16} \mathrm{NO}_{3} \mathrm{~F}_{3} \mathrm{Na}$ 350.0974; Found 350.0972.

Preparation of $\mathbf{3 g}$ under basic conditions: Following GP1, using quinoline-6-carboxylate (300 $\mathrm{mg}, 1.60 \mathrm{mmol}), \mathrm{BF}_{3} \cdot \mathrm{OEt}_{2}$ (250 $\mathrm{mg}, 1.76 \mathrm{mmol}$ ), isopropylmagnesium chloride lithium chloride complex solution ( $1.48 \mathrm{ml}, 1.3 \mathrm{M}$ in THF, $1.92 \mathrm{mmol})$, TFAA (1.01 g, $4.81 \mathrm{mmol})$ and TEA $(679 \mathrm{mg}, 4.81 \mathrm{mmol})$, the reaction mixture was stirred at r.t. overnight. Purification by flash chromatography (PE:EA = 100:1 10:1) furnishes the desired product (324 mg, 62\% yield) as a yellow solid, as well as the recovered quinoline-6-carboxylate $(92 \mathrm{mg})$, the yield based on the recovered starting material is $89 \%$.

(4-Cyclohexyl-1,4-dihydroquinolin-3-yl)-2,2,2-trifluoroethan-1one (3h): Following GP1, using quinoline (250 mg, $1.94 \mathrm{mmol})$, $\mathrm{BF}_{3} \cdot \mathrm{OEt}_{2}$ (302 mg, $2.13 \mathrm{mmol}$ ), cyclohexylmagnesium bromide 
solution (2.33 ml, $1.0 \mathrm{M}$ in THF, $2.33 \mathrm{mmol}$ ), TFAA $(815 \mathrm{mg}$, $3.88 \mathrm{mmol})$ and TFA $(1.327 \mathrm{~g}, 11.64 \mathrm{mmol})$, the reaction mixture was stirred at r.t. overnight. Purification by flash chromatography (PE:EA = 100:1 1:1) furnishes the desired product $(63 \mathrm{mg}, 11 \%$ yield) as a yellow solid, as well as the recovered quinoline (151 $\mathrm{mg}$ ), the yield based on the recovered starting material is $27 \% .{ }^{1} \mathrm{H}$ NMR $\left(400 \mathrm{MHz}, \mathrm{CDCl}_{3}\right) \delta 7.70(\mathrm{~d}, J=6.4 \mathrm{~Hz}, 1 \mathrm{H}), 7.20-7.27(\mathrm{~m}$, $1 \mathrm{H}), 7.10-7.19(\mathrm{~m}, 2 \mathrm{H}), 7.00(\mathrm{~s}, 1 \mathrm{H}), 6.80(\mathrm{~d}, J=7.6 \mathrm{~Hz}, 1 \mathrm{H})$, $4.00(\mathrm{~d}, J=4 \mathrm{~Hz}, 1 \mathrm{H}), 1.52-1.69(\mathrm{~m}, 4 \mathrm{H}), 1.26-1.43(\mathrm{~m}, 2 \mathrm{H})$, $1.04-1.15(\mathrm{~m}, 3 \mathrm{H}), 0.92-0.95(\mathrm{~m}, 1 \mathrm{H}), 0.60-0.64(\mathrm{~m}, 1 \mathrm{H}) .{ }^{13} \mathrm{C}\{1 \mathrm{H}\}$ NMR $\left(100 \mathrm{MHz}, \mathrm{CDCl}_{3}\right) \delta 177.0(\mathrm{q}, J=32 \mathrm{~Hz}), 143.5(\mathrm{q}, J=5$ $\mathrm{Hz}), 135.8,130.6,127.2,124.7,123.7,117.8(\mathrm{q}, J=290 \mathrm{~Hz})$, 115.1, 106.9, 45.9, 40.4, 29.9, 28.4, 26.6, 26.6, 26.4. HRMS (+ESI-TOF) $\mathrm{m} / \mathrm{z}:[\mathrm{M}+\mathrm{Na}]^{+}$Calcd for $\mathrm{C}_{17} \mathrm{H}_{18} \mathrm{NOF}_{3} \mathrm{Na} 332.1233$; Found 332.1227.

2,2,2-Trifluoro-1-(4-isopropyl-6-phenyl-1,4-dihydroquinolin-3yl)ethan-1-one (3i): Following GP1, using 6-phenylquinoline (410 mg, $2.00 \mathrm{mmol}$ ), $\mathrm{BF}_{3} \cdot \mathrm{OEt}_{2}$ (283 mg, $2.20 \mathrm{mmol}$ ), isopropylmagnesium chloride lithium chloride complex solution $(1.85 \mathrm{ml}$, $1.3 \mathrm{M}$ in THF, $2.40 \mathrm{mmol}$ ), TFAA (841 $\mathrm{mg}, 4.00 \mathrm{mmol}$ ) and TFA (684 $\mathrm{mg}, 6.00 \mathrm{mmol}$ ), the reaction mixture was stirred at r.t. overnight. Purification by flash chromatography (PE:EA = 100:1 20:1) furnishes the desired product $(458 \mathrm{mg}, 66 \%$ yield) as a yellow solid. ${ }^{1} \mathrm{H}$ NMR $\left(400 \mathrm{MHz}, \mathrm{CDCl}_{3}\right) \delta 7.71(\mathrm{~d}, J=6.4 \mathrm{~Hz}, 1 \mathrm{H}), 7.57$ $(\mathrm{d}, J=1.6 \mathrm{~Hz}, 2 \mathrm{H}), 7.42-7.55(\mathrm{~m}, 3 \mathrm{H}), 7.33-7.39(\mathrm{~m}, 2 \mathrm{H}), 6.88(\mathrm{~d}$, $J=8.0 \mathrm{~Hz}, 2 \mathrm{H}), 4.09(\mathrm{~d}, J=3.6 \mathrm{~Hz}, 1 \mathrm{H}), 1.84-1.92(\mathrm{~m}, 1 \mathrm{H}), 0.92$ $(\mathrm{d}, J=6.8 \mathrm{~Hz}, 3 \mathrm{H}), 0.71(\mathrm{~d}, J=7.2 \mathrm{~Hz}, 3 \mathrm{H}) .{ }^{13} \mathrm{C}\{1 \mathrm{H}\} \mathrm{NMR}(100$ $\left.\mathrm{MHz}, \mathrm{CDCl}_{3}\right) \delta 177.1(\mathrm{q}, J=33.0 \mathrm{~Hz}), 143.6(\mathrm{q}, J=5.0 \mathrm{~Hz})$, $140.4,138.0,135.1,129.2,129.0,127.4,126.9,126.1,123.6$, 117.6 (q, $J=341.0 \mathrm{~Hz}), 115.7,106.9,40.9,35.7,19.8,17.8$. HRMS (+ESI-TOF) m/z: $[\mathrm{M}+\mathrm{Na}]^{+}$Calcd for $\mathrm{C}_{20} \mathrm{H}_{18} \mathrm{NOF}_{3} \mathrm{Na}$ 368.1233; Found 368.1223.

Isopropyl-5-(2,2,2-trifluoroacetyl)-1,4-dihydropyridine-3carbonitrile (3j): Following GP1, using 3-cyanopyridine $(250 \mathrm{mg}$, $2.41 \mathrm{mmol}), \mathrm{BF}_{3} \cdot \mathrm{OEt}_{2}(375 \mathrm{mg}, 2.65 \mathrm{mmol}$ ), isopropylmagnesium chloride lithium chloride complex solution $(2.2 \mathrm{ml}, 1.3 \mathrm{M}$ in THF, $2.89 \mathrm{mmol})$, TFAA (1.0 g, $4.82 \mathrm{mmol})$ and TFA (821 mg, 7.23 mmol), the reaction mixture was stirred at r.t. overnight. Purification by flash chromatography (PE:EA $=50: 1 \sim 1: 1$ ) furnishes the desired product (336 mg, 62\% yield) as a yellow solid. ${ }^{1} \mathrm{H}$ NMR (400 MHz, d6-DMSO) $\delta 9.99$ (s, 1H), 7.65 (s, 1H), 7.38 (s, 1H), $3.46(\mathrm{~d}, J=3.2 \mathrm{~Hz}, 1 \mathrm{H}), 1.61-1.66(\mathrm{~m}, 1 \mathrm{H}), 0.91(\mathrm{~d}, J=7.2 \mathrm{~Hz}$, $3 \mathrm{H}), 0.78(\mathrm{~d}, J=6.8 \mathrm{~Hz}, 3 \mathrm{H}) .{ }^{13} \mathrm{C}\{1 \mathrm{H}\}$ NMR $(100 \mathrm{MHz}, \mathrm{d} 6-$ DMSO) $\delta 176.7(\mathrm{q}, J=33 \mathrm{~Hz}), 143.3(\mathrm{q}, J=5 \mathrm{~Hz}), 139.2,120.0$, $116.9(\mathrm{q}, J=290 \mathrm{~Hz}), 105.7,86.5,37.4,34.3,19.0,17.0$. HRMS (-ESI-TOF) $m / z$ : [M-H] Calcd for $\mathrm{C}_{11} \mathrm{H}_{10} \mathrm{~N}_{2} \mathrm{OF}_{3}$ 243.0751; Found 243.0761 .

Methyl 4-isopropyl-5-(2,2,2-trifluoroacetyl)-1,4dihydropyridine-3-carboxylate (3k): Following GP1, using methyl nicotinate (250 mg, $1.83 \mathrm{mmol}), \mathrm{BF}_{3} \cdot \mathrm{OEt}_{2}(284 \mathrm{mg}, 2.02 \mathrm{mmol}$ ), isopropylmagnesium chloride lithium chloride complex solution (1.7 ml, 1.3 M in THF, $2.20 \mathrm{mmol})$, TFAA (765 mg, $3.66 \mathrm{mmol})$ and TFA (1.24 g, $10.98 \mathrm{mmol})$, the reaction mixture was stirred at r.t. for $2.5 \mathrm{~h}$. Purification by flash chromatography (PE:EA = 50:1 1:1) furnishes the desired product $(160 \mathrm{mg}, 32 \%$ yield) as a yellow solid. ${ }^{1} \mathrm{H}$ NMR $\left(400 \mathrm{MHz}, \mathrm{CDCl}_{3}\right) \delta 8.03(\mathrm{~s}, 1 \mathrm{H}), 7.59(\mathrm{~d}$, $J=5.6 \mathrm{~Hz}, 1 \mathrm{H}), 7.40(\mathrm{~d}, J=4.8 \mathrm{~Hz}, 1 \mathrm{H}), 4.01(\mathrm{~d}, J=8.4 \mathrm{~Hz}, 1 \mathrm{H})$, $3.77(\mathrm{~s}, 3 \mathrm{H}), 1.66-1.76(\mathrm{~m}, 1 \mathrm{H}), 0.78(\mathrm{~d}, J=6.8 \mathrm{~Hz}, 6 \mathrm{H}) .{ }^{13} \mathrm{C}\{1 \mathrm{H}\}$ NMR $\left(100 \mathrm{MHz}, \mathrm{CDCl}_{3}\right) \delta 178.9(\mathrm{q}, J=33 \mathrm{~Hz}), 168.1,142.7(\mathrm{q}$, $J=5 \mathrm{~Hz}), 134.0,117.3(\mathrm{q}, J=290 \mathrm{~Hz}), 109.2,108.6,51.8,35.2$, 35.0, 18.5, 18.1. HRMS (-ESI-TOF) $\mathrm{m} / \mathrm{z}$ : [M-H] Calcd for $\mathrm{C}_{12} \mathrm{H}_{13} \mathrm{NO}_{3} \mathrm{~F}_{3} 276.0853$; Found 276.0852.

Ethyl 2-chloro-4-isopropyl-5-(2,2,2-trifluoroacetyl)-1,4dihydropyridine-3-carboxylate (3l): Following GP1, using ethyl 2chloronicotinate $(250 \mathrm{mg}, 1.35 \mathrm{mmol}), \mathrm{BF}_{3} \cdot \mathrm{OEt}_{2}(211 \mathrm{mg}, 1.49$ mmol), isopropylmagnesium chloride lithium chloride complex solution (1.2 ml, 1.3 M in THF, $1.62 \mathrm{mmol}$ ), TFAA (567 mg, 2.70 mmol) and TFA (462 mg, $4.05 \mathrm{mmol}$ ), the reaction mixture was stirred at r.t. overnight. Purification by flash chromatography (PE:EA = 50:1 1:1) furnishes the desired product $(320 \mathrm{mg}, 73 \%$ yield) as a light yellow solid. ${ }^{1} \mathrm{H} \mathrm{NMR}\left(400 \mathrm{MHz}, \mathrm{CDCl}_{3}\right) \delta 7.44$ $(\mathrm{d}, J=6.0 \mathrm{~Hz}, 1 \mathrm{H}), 6.84(\mathrm{~d}, J=3.6 \mathrm{~Hz}, 1 \mathrm{H}), 4.20-4.33(\mathrm{~m}, 2 \mathrm{H})$, 4.19 (d, $J=4.4 \mathrm{~Hz}, 1 \mathrm{H}), 1.69-1.77(\mathrm{~m}, 1 \mathrm{H}), 1.33(\mathrm{t}, J=7.2 \mathrm{~Hz}$, $3 \mathrm{H}), 0.79-0.81(\mathrm{~m}, 6 \mathrm{H}) .{ }^{13} \mathrm{C}\{1 \mathrm{H}\}$ NMR $\left(100 \mathrm{MHz}, \mathrm{CDCl}_{3}\right) \delta$ $178.5(\mathrm{q}, J=33 \mathrm{~Hz}), 166.6,141.8,132.9,117.1(\mathrm{q}, J=289 \mathrm{~Hz})$, 109.0, 104.7, 61.3, 39.0, 35.1, 18.3, 18.1, 14.2. HRMS (+ESI-TOF) $m / z:[\mathrm{M}+\mathrm{Na}]^{+}$Calcd for $\mathrm{C}_{13} \mathrm{H}_{15} \mathrm{NO}_{3} \mathrm{~F}_{3} \mathrm{ClNa}$ 348.0585; Found 348.0574 .

1-(5-Chloro-4-isopropyl-1,4-dihydropyridin-3-yl)-2,2,2trifluoroethan-1-one (3m): Following GP1, using 3-chloropyridine (250 mg, $2.20 \mathrm{mmol}$ ), $\mathrm{BF}_{3} \cdot \mathrm{OEt}_{2}$ (343 mg, $2.42 \mathrm{mmol}$ ), isopropylmagnesium chloride lithium chloride complex solution $(2.03 \mathrm{ml}$, $1.3 \mathrm{M}$ in THF, $2.64 \mathrm{mmol}$ ), TFAA (924 mg, $4.40 \mathrm{mmol}$ ) and TFA $(1.5 \mathrm{~g}, 13.20 \mathrm{mmol})$, the reaction mixture was stirred at r.t. overnight. Purification by flash chromatography (PE:EA = 30:1 3:1) furnishes the desired product $(282 \mathrm{mg}, 51 \%$ yield) as a yellow solid. ${ }^{1} \mathrm{H}$ NMR $\left(400 \mathrm{MHz}, \mathrm{CDCl}_{3}\right) \delta 7.50(\mathrm{~d}, J=6.0 \mathrm{~Hz}, 1 \mathrm{H}), 6.72$ (s, 1H), $6.42(\mathrm{~d}, J=4.8 \mathrm{~Hz}, 1 \mathrm{H}), 3.72(\mathrm{~d}, J=2.4 \mathrm{~Hz}, 1 \mathrm{H}), 1.84-$ $1.92(\mathrm{~m}, 1 \mathrm{H}), 0.99(\mathrm{~d}, J=7.2 \mathrm{~Hz}, 3 \mathrm{H}), 0.85(\mathrm{~d}, J=7.2 \mathrm{~Hz}, 3 \mathrm{H})$. ${ }^{13} \mathrm{C}\{1 \mathrm{H}\}$ NMR $\left(100 \mathrm{MHz}, \mathrm{CDCl}_{3}\right) \delta 178.0(\mathrm{q}, J=32 \mathrm{~Hz}), 142.6$ $(\mathrm{q}, J=5 \mathrm{~Hz}), 121.8,117.5(\mathrm{q}, J=290 \mathrm{~Hz}), 117.1,105.1,44.1$, 34.2, 19.9, 18.3. HRMS (-ESI-TOF) $\mathrm{m} / \mathrm{z}$ : $[\mathrm{M}-\mathrm{H}]^{-}$Calcd for $\mathrm{C}_{10} \mathrm{H}_{10} \mathrm{NOF}_{3} \mathrm{Cl} 252.0409$; Found 252.0421.

1-(5-Benzoyl-4-(o-tolyl)-1,4-dihydropyridin-3-yl)-2,2,2-

trifluoroethan-1-one (3n): Following GP2, using phenyl(pyridin3-yl)methanone (250 mg, $1.37 \mathrm{mmol}), \mathrm{BF}_{3} \cdot \mathrm{OEt}_{2}(213 \mathrm{mg}, 1.51$ mmol), $o$-tolylmagnesium bromide solution $(1.65 \mathrm{ml}, 1.0 \mathrm{M}$ in THF, $1.65 \mathrm{mmol}$ ), TFAA (576 mg, $2.74 \mathrm{mmol}$ ) and TFA (469 mg, $4.11 \mathrm{mmol}$ ), the reaction mixture was stirred at r.t. overnight. Purification by flash chromatography (PE:EA $=50: 1 \sim 1: 1$ ) furnishes the desired product (113 mg, $22 \%$ yield) as a yellow solid. ${ }^{1} \mathrm{H}$ NMR (400 MHz, CD $\left.{ }_{3} \mathrm{OD}\right) \delta 9.49$ (s, 1H), 7.66 (s, 1H), 7.49$7.50(\mathrm{~m}, 1 \mathrm{H}), 7.37-7.48(\mathrm{~m}, 4 \mathrm{H}), 7.30(\mathrm{~d}, J=7.6 \mathrm{~Hz}, 1 \mathrm{H}), 7.11-$ $7.15(\mathrm{~m}, 1 \mathrm{H}), 7.00-7.04(\mathrm{~m}, 3 \mathrm{H}), 5.38(\mathrm{~s}, 1 \mathrm{H}), 2.75(\mathrm{~s}, 3 \mathrm{H})$ ${ }^{13} \mathrm{C}\{1 \mathrm{H}\}$ NMR $\left(100 \mathrm{MHz}, \mathrm{CD}_{3} \mathrm{OD}\right) \delta 197.5,178.8(\mathrm{q}, J=33 \mathrm{~Hz})$, $146.8,142.0$ (q, $J=4 \mathrm{~Hz}), 140.1,138.4,137.3,132.5,130.7$, $129.8,129.43,129.42,127.6,127.5,122.1,118.5(\mathrm{q}, J=289 \mathrm{~Hz})$, 114.3, 33.4, 20.1. HRMS (+ESI-TOF) $\mathrm{m} / \mathrm{z}:[\mathrm{M}+\mathrm{Na}]^{+}$Calcd for $\mathrm{C}_{21} \mathrm{H}_{16} \mathrm{NO}_{2} \mathrm{~F}_{3} \mathrm{Na}$ 394.1025; Found 394.1018.

4-(Prop-1-en-2-yl)-5-(2,2,2-trifluoroacetyl)-1,4-

dihydropyridine-3-carbonitrile (3o): Following GP2, using 3cyanopyridine $(200 \mathrm{mg}, 1.91 \mathrm{mmol}), \mathrm{BF}_{3} \cdot \mathrm{OEt}_{2}(298 \mathrm{mg}, 2.10$ mmol), isopropenylmagnesium bromide solution $(2.29 \mathrm{ml}, 1.0 \mathrm{M}$ in THF, $2.29 \mathrm{mmol})$, TFAA (801 $\mathrm{mg}, 3.81 \mathrm{mmol})$ and TFA (1.30 $\mathrm{g}, 11.44 \mathrm{mmol}$ ), the reaction mixture was stirred at r.t. overnight. Purification by flash chromatography (PE:EA $=80: 1 \sim 10: 1$ ) furnishes the desired product (108 $\mathrm{mg}, 23 \%$ yield) as a yellow solid, as well as the recovered 3-cyanopyridine $(48 \mathrm{mg})$, the yield based on the recovered starting material is $53 \%$. ${ }^{1} \mathrm{H} \mathrm{NMR}(400 \mathrm{MHz}$, $\left.\mathrm{CD}_{3} \mathrm{OD}\right) \delta 7.56(\mathrm{~s}, 1 \mathrm{H}), 7.06(\mathrm{~s}, 1 \mathrm{H}), 4.88(\mathrm{~d}, J=2.8 \mathrm{~Hz}, 2 \mathrm{H})$, $4.13(\mathrm{~s}, 1 \mathrm{H}), 1.79(\mathrm{~s}, 3 \mathrm{H}) .{ }^{13} \mathrm{C}\{1 \mathrm{H}\}$ NMR $\left(100 \mathrm{MHz}, \mathrm{CD}_{3} \mathrm{OD}\right) \delta$ 178.9 (q, $J=33 \mathrm{~Hz}), 147.2,142.9(\mathrm{q}, J=5 \mathrm{~Hz}), 119.8,118.5$ (q, $J$ $=289 \mathrm{~Hz}), 114.3,108.4,92.2,41.6,20.0$. HRMS (-ESI-TOF) m/z: [M-H] Calcd for $\mathrm{C}_{11} \mathrm{H}_{8} \mathrm{~N}_{2} \mathrm{OF}_{3}$ 241.0594; Found 241.0593.

4-(O-tolyl)-5-(2,2,2-trifluoroacetyl)-1,4-dihydropyridine-3carbonitrile (3p): Following GP2, using 3-cyanopyridine (1.0 g, $9.61 \mathrm{mmol}), \mathrm{BF}_{3} \cdot \mathrm{OEt}_{2}(1.5 \mathrm{~g}, 10.57 \mathrm{mmol}), o$-tolylmagnesium bromide solution (14.4 ml, $0.8 \mathrm{M}$ in THF, $11.53 \mathrm{mmol}$ ), TFAA (4.036 g, $19.28 \mathrm{mmol})$ and TFA (3.287 g, $28.92 \mathrm{mmol})$, the reaction mixture was stirred at r.t. overnight. Purification by flash chromatography (PE:EA = 50:1 5:1) furnishes the desired product (2.201 g, 78\% yield) as a yellow solid. ${ }^{1} \mathrm{H}$ NMR $(400 \mathrm{MHz}, \mathrm{d} 6-$ 
DMSO) $\delta 10.08(\mathrm{~s}, 1 \mathrm{H}), 7.74(\mathrm{~d}, J=5.6 \mathrm{~Hz}, 1 \mathrm{H}), 7.24(\mathrm{~d}, J=4.8$ $\mathrm{Hz}, 1 \mathrm{H}), 7.18-7.21(\mathrm{~m}, 2 \mathrm{H}), 7.12-7.17(\mathrm{~m}, 2 \mathrm{H}), 4.86(\mathrm{~s}, 1 \mathrm{H}), 2.50$ (s, 3H). ${ }^{13} \mathrm{C}\{1 \mathrm{H}\}$ NMR (100 MHz, d6-DMSO) $\delta 176.2$ (q, $J=33$ $\mathrm{Hz}), 143.8,142.0(\mathrm{q}, J=5 \mathrm{~Hz}), 135.7,134.6,129.8,128.4,127.1$, 127.0, 118.7, 116.7 (q, $J=290 \mathrm{~Hz}$ ), 108.3, 92.0, 33.5, 19.0. HRMS (-ESI-TOF) $\mathrm{m} / \mathrm{z}:$ [M-H] $]^{-}$Calcd for $\mathrm{C}_{15} \mathrm{H}_{10} \mathrm{~N}_{2} \mathrm{OF}_{3} 291.0751$; Found 291.0762.

4-Phenyl-5-(2,2,2-trifluoroacetyl)-1,4-dihydropyridine-3carbonitrile (3q): Following GP2, using 3-cyanopyridine (250 mg, $2.41 \mathrm{mmol}), \mathrm{BF}_{3} \cdot \mathrm{OEt}_{2}(375 \mathrm{mg}, 2.65 \mathrm{mmol})$, phenylmagnesium bromide solution (2.89 ml, $1 \mathrm{M}$ in THF, $2.89 \mathrm{mmol})$, TFAA (1.0 g, $4.82 \mathrm{mmol}$ ) and TFA (821 $\mathrm{mg}, 7.23 \mathrm{mmol})$, the reaction mixture was stirred at r.t. overnight. Purification by flash chromatography (PE:EA = 50:1 2:1) furnishes the desired product (312 mg, 47\% yield) as a yellow solid. ${ }^{1} \mathrm{H}$ NMR (400 MHz, d6-DMSO) $\delta 10.12$ $(\mathrm{s}, 1 \mathrm{H}), 7.73(\mathrm{~d}, J=5.2 \mathrm{~Hz}, 1 \mathrm{H}), 7.34-7.37(\mathrm{~m}, 2 \mathrm{H}), 7.25-7.30(\mathrm{~m}$, $4 \mathrm{H}), 4.59(\mathrm{~s}, 1 \mathrm{H}) .{ }^{13} \mathrm{C}\{1 \mathrm{H}\} \mathrm{NMR}(100 \mathrm{MHz}, \mathrm{d} 6-\mathrm{DMSO}) \delta 176.2$ $(\mathrm{q}, J=33 \mathrm{~Hz}), 144.2,142.0(\mathrm{q}, J=4 \mathrm{~Hz}), 136.1,128.7,127.4$, 118.6, $116.7(\mathrm{q}, J=290 \mathrm{~Hz}), 107.1,91.8,37.9$. HRMS (-ESI-TOF) $m / z:[\mathrm{M}-\mathrm{H}]^{-}$Calcd for $\mathrm{C}_{14} \mathrm{H}_{8} \mathrm{~N}_{2} \mathrm{OF}_{3}$ 277.0594; Found 277.0592.

4-(P-tolyl)-5-(2,2,2-trifluoroacetyl)-1,4-dihydropyridine-3carbonitrile (3r): Following GP2, using 3-cyanopyridine (250 mg, $2.41 \mathrm{mmol}$ ), $\mathrm{BF}_{3} \cdot \mathrm{OEt}_{2}(375 \mathrm{mg}, 2.65 \mathrm{mmol}), p$-tolylmagnesium bromide solution ( $2.89 \mathrm{ml}, 1 \mathrm{M}$ in THF, $2.89 \mathrm{mmol})$, TFAA (1.0 g, $4.82 \mathrm{mmol})$ and TFA (821 $\mathrm{mg}, 7.23 \mathrm{mmol})$, the reaction mixture was stirred at r.t. overnight. Purification by flash chromatography (PE:EA = 50:1 2:1) furnishes the desired product $(421 \mathrm{mg}, 60 \%$ yield) as a yellow solid, as well as the recovered 3-cyanopyridine $(53 \mathrm{mg})$, the yield based on the recovered starting material is $75 \%$. ${ }^{1} \mathrm{H}$ NMR (400 MHz, CD $\left.{ }_{3} \mathrm{OD}\right) \delta 7.62(\mathrm{~s}, 1 \mathrm{H}), 7.15(\mathrm{q}, J=8 \mathrm{~Hz}$, $4 \mathrm{H}), 7.04(\mathrm{~s}, 1 \mathrm{H}), 4.58(\mathrm{~s}, 1 \mathrm{H}), 2.30(\mathrm{~s}, 3 \mathrm{H}) .{ }^{13} \mathrm{C}\{1 \mathrm{H}\}$ NMR $(100$ $\left.\mathrm{MHz}, \mathrm{CD}_{3} \mathrm{OD}\right) \delta 178.6(\mathrm{q}, J=33 \mathrm{~Hz}), 142.6,142.1(\mathrm{q}, J=5 \mathrm{~Hz})$, 138.4, 136.5, 130.3, 128.7, 119.6, 118.2 (q, $J=289 \mathrm{~Hz}), 109.8$, 93.9, 39.4, 21.1. HRMS (-ESI-TOF) $\mathrm{m} / \mathrm{z}$ : [M-H] Calcd for $\mathrm{C}_{15} \mathrm{H}_{10} \mathrm{~N}_{2} \mathrm{OF}_{3}$ 291.0751; Found 291.0744.

4-Mesityl-5-(2,2,2-trifluoroacetyl)-1,4-dihydropyridine-3carbonitrile (3s): Following GP2, using 3-cyanopyridine (200 mg, $1.91 \mathrm{mmol}), \mathrm{BF}_{3} \cdot \mathrm{OEt}_{2}(298 \mathrm{mg}, 2.10 \mathrm{mmol}), 2,4,6-$ trimethylphenylmagnesium bromide solution $(2.29 \mathrm{ml}, 1.0 \mathrm{M}$ in THF, $2.29 \mathrm{mmol})$, TFAA (801 mg, $3.81 \mathrm{mmol})$ and TFA (1.30 g, $11.44 \mathrm{mmol}$ ), the reaction mixture was stirred at r.t. overnight. Purification by flash chromatography (PE:EA $=80: 1 \sim 10: 1$ ) furnishes the desired product ( $245 \mathrm{mg}, 40 \%$ yield) as a yellow solid, as well as the recovered 3-cyanopyridine (109 $\mathrm{mg})$, the yield based on the recovered starting material is $80 \%$. ${ }^{1} \mathrm{H}$ NMR $(400$ MHz, d6-DMSO) $\delta 9.98$ (s, 1H), 7.53 (s, 1H), 7.19 (s, 1H), 6.82 $(\mathrm{d}, J=3.6 \mathrm{~Hz}, 2 \mathrm{H}), 5.23(\mathrm{~s}, 1 \mathrm{H}), 2.45(\mathrm{~s}, 3 \mathrm{H}), 2.52(\mathrm{~s}, 3 \mathrm{H}), 2.18(\mathrm{~s}$, $3 \mathrm{H}) .{ }^{13} \mathrm{C}\{1 \mathrm{H}\}$ NMR $(100 \mathrm{MHz}, \mathrm{d} 6-\mathrm{DMSO}) \delta 176.1$ (q, $J=32.0$ $\mathrm{Hz}), 142.6$ (q, $J=3.0 \mathrm{~Hz}), 137.3,136.7,136.4,135.8,135.5$, $131.3,128.9,118.3,116.7(\mathrm{q}, J=291.0 \mathrm{~Hz}), 106.7,89.9,2.9,20.8$, 20.3, 18.4. HRMS (+ESI-TOF) $\mathrm{m} / \mathrm{z}: \quad[\mathrm{M}+\mathrm{Na}]^{+}$Calcd for $\mathrm{C}_{17} \mathrm{H}_{15} \mathrm{~N}_{2} \mathrm{OF}_{3} \mathrm{Na}$ 343.1029; Found 343.1027.

4-(3-Methoxyphenyl)-5-(2,2,2-trifluoroacetyl)-1,4-

dihydropyridine-3-carbonitrile (3t): Following GP2, using 3cyanopyridine (200 mg, $1.91 \mathrm{mmol}), \mathrm{BF}_{3} \cdot \mathrm{OEt}_{2}(298 \mathrm{mg}, 2.10$ mmol), 3-methoxyphenylmagnesium bromide solution $(2.29 \mathrm{ml}$, $1.0 \mathrm{M}$ in THF, $2.29 \mathrm{mmol})$, TFAA (801 $\mathrm{mg}, 3.81 \mathrm{mmol})$ and TFA $(1.30 \mathrm{~g}, 11.44 \mathrm{mmol})$, the reaction mixture was stirred at r.t. overnight. Purification by flash chromatography (PE:EA = 50:1 10:1) furnishes the desired product (294 $\mathrm{mg}, 50 \%$ yield) as a yellow solid, as well as the recovered 3-cyanopyridine $(65 \mathrm{mg})$, the yield based on the recovered starting material is $74 \% .{ }^{1} \mathrm{H}$ NMR $(400$ $\left.\mathrm{MHz}, \mathrm{CD}_{3} \mathrm{OD}\right) \delta 7.65(\mathrm{~s}, 1 \mathrm{H}), 7.24(\mathrm{t}, J=8.0 \mathrm{~Hz}, 1 \mathrm{H}), 7.05(\mathrm{~s}$, $1 \mathrm{H}), 6.81-6.88(\mathrm{~m}, 3 \mathrm{H}), 4.61(\mathrm{~s}, 1 \mathrm{H}), 3.78(\mathrm{~s}, 3 \mathrm{H}) .{ }^{13} \mathrm{C}\{1 \mathrm{H}\} \mathrm{NMR}$ $\left(100 \mathrm{MHz}, \mathrm{CD}_{3} \mathrm{OD}\right) \delta 178.6(\mathrm{q}, J=34.0 \mathrm{~Hz}), 161.4,146.8,142.2$ $(\mathrm{q}, J=5.0 \mathrm{~Hz}), 136.6,130.7,121.0,119.5,118.3(\mathrm{q}, J=290.0 \mathrm{~Hz})$,
114.7, 113.8, 109.5, 93.7, 55.6, 39.8. HRMS (+ESI-TOF) $\mathrm{m} / \mathrm{z}$ : $[\mathrm{M}+\mathrm{Na}]^{+}$Calcd for $\mathrm{C}_{15} \mathrm{H}_{11} \mathrm{~N}_{2} \mathrm{O}_{2} \mathrm{~F}_{3} \mathrm{Na}$ 331.0665; Found 331.0662.

4-(4-Methoxyphenyl)-5-(2,2,2-trifluoroacetyl)-1,4-

dihydropyridine-3-carbonitrile $(\mathbf{3 u})$ : Following GP2, using 3cyanopyridine (250 mg, $2.40 \mathrm{mmol}$ ), $\mathrm{BF}_{3} \cdot \mathrm{OEt}_{2}(369 \mathrm{mg}, 2.60$ mmol), 4-methoxyphenylmagnesium bromide solution $(3.60 \mathrm{ml}$, $1.0 \mathrm{M}$ in THF, $3.60 \mathrm{mmol})$, TFAA $(1.01 \mathrm{~g}, 4.80 \mathrm{mmol})$ and TFA (821 mg, $7.20 \mathrm{mmol}$ ), the reaction mixture was stirred at r.t. overnight. Purification by flash chromatography (PE:EA = 100:1 10:1) furnishes the desired product ( $269 \mathrm{mg}, 36 \%$ yield) as a yellow solid, as well as the recovered 3-cyanopyridine $(130 \mathrm{mg})$, the yield based on the recovered starting material is $70 \%$. ${ }^{1} \mathrm{H}$ NMR $(400$ $\left.\mathrm{MHz}, \mathrm{CD}_{3} \mathrm{OD}\right) \delta 7.61(\mathrm{~s}, 1 \mathrm{H}), 7.21(\mathrm{~d}, J=8.4 \mathrm{~Hz}, 2 \mathrm{H}), 7.05(\mathrm{~s}$, $1 \mathrm{H}), 6.88(\mathrm{~d}, J=8.8 \mathrm{~Hz}, 2 \mathrm{H}), 4.57(\mathrm{~s}, 1 \mathrm{H}), 3.78(\mathrm{~s}, 3 \mathrm{H}) .{ }^{13} \mathrm{C}\{1 \mathrm{H}\}$ NMR (100 MHz, d6-DMSO) $\delta 176.2(\mathrm{q}, J=33.0 \mathrm{~Hz}), 158.5$, $141.6(\mathrm{q}, J=5.0 \mathrm{~Hz}), 136.5,135.8,128.5,118.7,116.7$ (q, $J=$ $290.0 \mathrm{~Hz}), 114.0,107.4,92.0,55.0,37.0$. HRMS (-ESI-TOF) $\mathrm{m} / \mathrm{z}$ : [M-H] Calcd for $\mathrm{C}_{15} \mathrm{H}_{10} \mathrm{~N}_{2} \mathrm{O}_{2} \mathrm{~F}_{3}$ 307.0700; Found 307.0694.

4-(3-Fluorophenyl)-5-(2,2,2-trifluoroacetyl)-1,4-

dihydropyridine-3-carbonitrile (3v): Following GP2, using 3cyanopyridine $(250 \mathrm{mg}, 2.41 \mathrm{mmol}), \mathrm{BF}_{3} \cdot \mathrm{OEt}_{2}(375 \mathrm{mg}, 2.65$ mmol), 3-fluorophenylmagnesium bromide solution (2.89 ml, 1.0 $\mathrm{M}$ in THF, $2.89 \mathrm{mmol})$, TFAA (1.0 g, $4.82 \mathrm{mmol})$ and TFA (825 $\mathrm{mg}, 7.23 \mathrm{mmol})$, the reaction mixture was stirred at r.t. overnight. Purification by flash chromatography (PE:EA = 50:1 2:1) furnishes the desired product (563 mg, 79\% yield) as a yellow solid. ${ }^{1} \mathrm{H}$ NMR (400 MHz, CD $\left.\mathrm{OD}\right) \delta 9.72$ (s, 1H), 7.67 (s, 1H), 7.32$7.37(\mathrm{~m}, 1 \mathrm{H}), 7.14(\mathrm{~d}, J=8 \mathrm{~Hz}, 1 \mathrm{H}), 7.09-7.10(\mathrm{~m}, 1 \mathrm{H}), 6.96-7.04$ $(\mathrm{m}, 2 \mathrm{H}), 4.68(\mathrm{~s}, 1 \mathrm{H}),{ }^{13} \mathrm{C}\{1 \mathrm{H}\} \mathrm{NMR}\left(100 \mathrm{MHz}, \mathrm{CD}_{3} \mathrm{OD}\right) \delta 178.6$ (q, $J=34 \mathrm{~Hz}), 164.4$ (d, $J=244 \mathrm{~Hz}), 147.8$ (d, $J=6 \mathrm{~Hz}), 142.4$ (d, $J=5 \mathrm{~Hz}), 137.0,131.3(\mathrm{~d}, J=8 \mathrm{~Hz}), 124.6(\mathrm{~d}, J=3 \mathrm{~Hz}), 119.4$, $118.2(\mathrm{q}, J=289 \mathrm{~Hz}), 115.4(\mathrm{q}, J=44 \mathrm{~Hz}), 115.4,109.1,93.1$, 39.5. HRMS (-ESI-TOF) $m / z$ : $[\mathrm{M}-\mathrm{H}]^{-}$Calcd for $\mathrm{C}_{14} \mathrm{H}_{7} \mathrm{~N}_{2} \mathrm{OF}_{4}$ 295.0500; Found 295.0486.

2,2,2-Trichloro-1-(4-isopropyl-1,4-dihydroquinolin-3-yl)ethan1-one (4): Following GP1, using quinoline $(250 \mathrm{mg}, 1.94 \mathrm{mmol})$, $\mathrm{BF}_{3} \cdot \mathrm{OEt}_{2}$ (302 mg, $2.13 \mathrm{mmol}$ ), isopropylmagnesium chloride lithium chloride complex solution $(1.8 \mathrm{ml}, 1.3 \mathrm{M}$ in THF, 2.33 mmol), trichloroacetic anhydride $(1.198 \mathrm{~g}, 3.88 \mathrm{mmol})$ and TFA (1.327 $\mathrm{g}, 11.64 \mathrm{mmol})$, the reaction mixture was stirred at r.t. overnight. Purification by flash chromatography (PE:EA = 100:1 5:1) furnishes the desired product ( $278 \mathrm{mg}, 45 \%$ yield) as a yellow solid, as well as the recovered quinoline $(75 \mathrm{mg})$, the yield based on the recovered starting material is $65 \%$. ${ }^{1} \mathrm{H}$ NMR $(400$ MHz, d6-DMSO) $\delta 10.13(\mathrm{~d}, J=6.4 \mathrm{~Hz}, 1 \mathrm{H}), 8.17(\mathrm{~d}, J=6.4 \mathrm{~Hz}$, $1 \mathrm{H}), 7.20-7.24(\mathrm{~m}, 1 \mathrm{H}), 7.10-7.20(\mathrm{~m}, 1 \mathrm{H}), 7.07-7.09(\mathrm{~m}, 1 \mathrm{H})$, $7.01(\mathrm{~d}, J=7.6 \mathrm{~Hz}, 1 \mathrm{H}), 3.88(\mathrm{~d}, J=4 \mathrm{~Hz}, 1 \mathrm{H}), 1.63-1.71(\mathrm{~m}, 1 \mathrm{H})$, $0.79(\mathrm{~d}, J=6.8 \mathrm{~Hz}, 3 \mathrm{H}), 0.60(\mathrm{~d}, J=7.2 \mathrm{~Hz}, 3 \mathrm{H}) .{ }^{13} \mathrm{C}\{1 \mathrm{H}\} \mathrm{NMR}$ (100 MHz, d6-DMSO) $\delta 177.9,144.1,136.0,129.8,127.2,123.9$, 122.6, 115.5, 98.2, 96.8, 41.2, 35.2, 19.2, 17.6. HRMS (+ESI-TOF) $m / z:[\mathrm{M}+\mathrm{Na}]^{+}$Calcd for $\mathrm{C}_{14} \mathrm{H}_{14} \mathrm{NOCl}_{3} \mathrm{Na}$ 340.0033; Found 340.0030 .

Preparation of 5 and 6: A dry and argon flushed $25 \mathrm{ml}$ flask, equipped with a magnetic stirring bar and a rubber septum is charged with a solution of quinoline $(250 \mathrm{mg}, 1.94 \mathrm{mmol})$ in dry THF $(3.9 \mathrm{ml})$ and cooled to $0{ }^{\circ} \mathrm{C} . \mathrm{BF}_{3} \cdot \mathrm{OEt}_{2}(302 \mathrm{mg}, 2.13 \mathrm{mmol})$ is added dropwise and stirred for $15 \mathrm{~min}$ at the same temperature. The reaction mixture is cooled to $-50{ }^{\circ} \mathrm{C}$ followed by dropwise addition of isopropylmagnesium chloride lithium chloride complex solution (1.8 $\mathrm{ml}, 1.3 \mathrm{M}$ in THF, $2.33 \mathrm{mmol})$, and stirring the reaction mixture at the same temperature for $30 \mathrm{~min}$. Then acetic anhydride (792 mg, $7.76 \mathrm{mmol})$ and $\mathrm{Et}_{3} \mathrm{~N}$ (589 mg, $\left.5.82 \mathrm{mmol}\right)$ are added, and the reaction mixture is increased to r.t. by removing the EA/dry ice bath. Stirring is continued at r.t. overnight. Finally, it is quenched with water and extracted with EA three times. The combined organic phases are washed with brine, dried 
over $\mathrm{Na}_{2} \mathrm{SO}_{4}$, and concentrated in vacuo to give the crude product. Purification by flash chromatography (PE:EA $=20: 1 \sim 2: 1$ ) furnishes the desired product $5(82 \mathrm{mg}, 18 \%)$ and $\mathbf{6}(88 \mathrm{mg}, 18 \%)$.

(4-Isopropyl-1,4-dihydroquinolin-3-yl)ethan-1-one $\quad(5): \quad{ }^{1} \mathrm{H}$ NMR (400 MHz, $\left.\mathrm{CDCl}_{3}\right) \delta 7.48(\mathrm{~d}, J=6.0 \mathrm{~Hz}, 1 \mathrm{H}), 7.10-7.15(\mathrm{~m}$, $2 \mathrm{H}), 6.99-7.03(\mathrm{~m}, 1 \mathrm{H}), 6.73(\mathrm{~d}, J=8.0 \mathrm{~Hz}, 1 \mathrm{H}), 6.35(\mathrm{~s}, 1 \mathrm{H})$, $4.02(\mathrm{~d}, J=4.0 \mathrm{~Hz}, 1 \mathrm{H}), 2.28(\mathrm{~s}, 3 \mathrm{H}), 1.73-1.81(\mathrm{~m}, 1 \mathrm{H}), 0.86(\mathrm{~d}$, $J=6.8 \mathrm{~Hz}, 3 \mathrm{H}), 0.63(\mathrm{~d}, J=6.8 \mathrm{~Hz}, 3 \mathrm{H}) .{ }^{13} \mathrm{C}\{1 \mathrm{H}\}$ NMR $(100$ $\left.\mathrm{MHz}, \mathrm{CDCl}_{3}\right) \delta 195.2,139.8,137.5,130.6,126.8,123.2,123.1$, $114.6,113.5,40.9,35.7,24.7,20.0,17.7$. HRMS (-ESI-TOF) $\mathrm{m} / \mathrm{z}$ : [M-H] Calcd for $\mathrm{C}_{14} \mathrm{H}_{16} \mathrm{NO} 214.1237$; Found 214.1235.

1,1'-(4-Isopropylquinoline-1,3(4H)-diyl)bis(ethan-1-one) (6): ${ }^{1} \mathrm{H}$ NMR (400 MHz, d6-DMSO) $\delta 8.17(\mathrm{~s}, 1 \mathrm{H}), 7.84(\mathrm{~d}, J=8 \mathrm{~Hz}$, $1 \mathrm{H}), 7.21-7.28(\mathrm{~m}, 3 \mathrm{H}), 3.76(\mathrm{~d}, J=5.2 \mathrm{~Hz}, 1 \mathrm{H}), 2.53(\mathrm{~s}, 3 \mathrm{H})$, $2.41(\mathrm{~s}, 3 \mathrm{H}), 1.64-1.69(\mathrm{~m}, 1 \mathrm{H}), 0.78(\mathrm{~d}, J=6.8 \mathrm{~Hz}, 3 \mathrm{H}), 0.59(\mathrm{~d}$ $J=6.8 \mathrm{~Hz}, 3 \mathrm{H}) .{ }^{13} \mathrm{C}\{1 \mathrm{H}\} \mathrm{NMR}(100 \mathrm{MHz}, \mathrm{d} 6-\mathrm{DMSO}) \delta 195.7$, $168.9,138.5,135.9,130.3,129.7,126.1,125.4,125.3,122.2,41.7$, 33.6, 25.5, 23.9, 19.7, 18.2. HRMS (+ESI-TOF) $\mathrm{m} / \mathrm{z}:[\mathrm{M}+\mathrm{H}]^{+}$ Calcd for $\mathrm{C}_{16} \mathrm{H}_{20} \mathrm{NO}_{2}$ 258.1489; Found 258.1473.

1-(4-Isopropylquinolin-1(4H)-yl)ethan-1-one (7): Following GP1, using quinoline (1 g, $7.74 \mathrm{mmol}), \mathrm{BF}_{3} \cdot \mathrm{OEt}_{2}(1.2 \mathrm{~g}, 8.54$ mmol), isopropylmagnesium chloride lithium chloride complex solution (7.2 ml, 1.3 M in THF, $9.32 \mathrm{mmol})$, acetic anhydride $(1.58 \mathrm{~g}, 15.52 \mathrm{mmol})$, TFA $(2.65 \mathrm{~g}, 23.28 \mathrm{mmol})$, the reaction mixture was stirred at r.t. overnight. Purification by flash chromatography (PE:EA = 100:1 1:1) furnishes the desired product $(230$ $\mathrm{mg}, 14 \%$ yield) as a yellow oil. ${ }^{1} \mathrm{H}$ NMR $\left(400 \mathrm{MHz}, \mathrm{CDCl}_{3}\right) \delta$ $7.82(\mathrm{~s}, 1 \mathrm{H}), 7.21-7.24(\mathrm{~m}, 1 \mathrm{H}), 7.14-7.18(\mathrm{~m}, 1 \mathrm{H}), 7.08-7.10(\mathrm{~m}$, $1 \mathrm{H}), 6.94-6.95(\mathrm{~m}, 1 \mathrm{H}), 5.49$ (dd, $J=6.0,7.2 \mathrm{~Hz}, 1 \mathrm{H}), 3.20$ (t, $J=$ $6.0 \mathrm{~Hz}, 1 \mathrm{H}), 2.36(\mathrm{~s}, 3 \mathrm{H}), 1.83-1.86(\mathrm{~m}, 1 \mathrm{H}), 0.90(\mathrm{~d}, J=6.8 \mathrm{~Hz}$, $3 \mathrm{H}), 0.83(\mathrm{~d}, J=6.8 \mathrm{~Hz}, 3 \mathrm{H}) .{ }^{13} \mathrm{C}\{1 \mathrm{H}\} \mathrm{NMR}\left(100 \mathrm{MHz}, \mathrm{CDCl}_{3}\right)$ $\delta 168.3,137.2,132.3,129.1,127.7,126.1,125.3,123.0,115.0$, 45.4, 35.4, 24.2, 20.0, 18.5. HRMS (+ESI-TOF) $\mathrm{m} / \mathrm{z}:[\mathrm{M}+\mathrm{Na}]^{+}$ Calcd for $\mathrm{C}_{14} \mathrm{H}_{17} \mathrm{NONa} 238.1202$; Found 238.1193.

1-(1-Benzoyl-4-isopropyl-1,4-dihydroquinolin-3-yl)-2,2,2trifluoroethan-1-one (9): To a solution of $\mathbf{3 a}(300 \mathrm{mg}, 1.11 \mathrm{mmol})$ in dry DCM $(2.2 \mathrm{ml})$ was added benzoyl chloride $(469 \mathrm{mg}, 3.33$ mmol) dropwise, followed by DMAP (27 mg, $0.22 \mathrm{mmol})$ and triethylamine (336 mg, $3.33 \mathrm{mmol}$ ). The reaction mixture was stirred at r.t. overnight. Then it is quenched with water and extracted with EA three times. The combined organic phases were washed with brine, dried over $\mathrm{Na}_{2} \mathrm{SO}_{4}$, and concentrated in vacuo to give the crude product. Purification by flash chromatography (PE:EA $=50: 1 \sim 1: 1$ ) furnishes the desired product $(348 \mathrm{mg}, 84 \%$ yield) as a yellow solid. ${ }^{1} \mathrm{H}$ NMR $\left(400 \mathrm{MHz}, \mathrm{CDCl}_{3}\right) \delta 8.56(\mathrm{~s}$, $1 \mathrm{H}), 7.67(\mathrm{~d}, J=7.6 \mathrm{~Hz}, 1 \mathrm{H}), 7.57-7.61(\mathrm{~m}, 1 \mathrm{H}), 7.45-7.48(\mathrm{~m}$, $2 \mathrm{H}), 7.17-7.24(\mathrm{~m}, 2 \mathrm{H}), 7.06-7.14(\mathrm{~m}, 2 \mathrm{H}), 3.91(\mathrm{~d}, J=6 \mathrm{~Hz}, 1 \mathrm{H})$, $1.86-1.94(\mathrm{~m}, 1 \mathrm{H}), 1.01(\mathrm{~d}, J=6.8 \mathrm{~Hz}, 3 \mathrm{H}), 0.89(\mathrm{~d}, J=6.8 \mathrm{~Hz}$, $3 \mathrm{H}) .{ }^{13} \mathrm{C}\{1 \mathrm{H}\} \mathrm{NMR}\left(100 \mathrm{MHz}, \mathrm{CDCl}_{3}\right) \delta 179.0(\mathrm{q}, J=34 \mathrm{~Hz})$, 169.0, 142.4 (q, $J=5 \mathrm{~Hz}), 136.3,133.6,133.0,130.5,129.3,129.0$, 128.4, 126.7 , 126.0, 121.6, 117.1, 116.9 (q, $J=290 \mathrm{~Hz}), 42.9$, 34.4, 20.4, 19.2. HRMS (+ESI-TOF) $\mathrm{m} / \mathrm{z}:[\mathrm{M}+\mathrm{H}]^{+}$Calcd for $\mathrm{C}_{21} \mathrm{H}_{19} \mathrm{~F}_{3} \mathrm{NO}_{2}$ 374.1362; Found 374.1354.

1-(1-Acetyl-4-isopropyl-1,4-dihydroquinolin-3-yl)-2,2,2-

trifluoroethan-1-one (10): To a solution of $\mathbf{3 a}(1 \mathrm{~g}, 3.70 \mathrm{mmol})$ in dry DCM $(7.4 \mathrm{ml})$ was added acetyl chloride $(872 \mathrm{mg}, 11.10$ mmol) dropwise, followed by DMAP (91 $\mathrm{mg}, 0.74 \mathrm{mmol})$ and triethylamine $(1.12 \mathrm{~g}, 11.10 \mathrm{mmol})$. The reaction mixture was stirred at r.t. overnight. Then it is quenched with water and extracted with EA three times. The combined organic phases were washed with brine, dried over $\mathrm{Na}_{2} \mathrm{SO}_{4}$, and concentrated in vacuo to give the crude product. Purification by flash chromatography (PE:EA = 50:1 5:1) furnishes the desired product $(1.02 \mathrm{~g}, 89 \%$ yield) as a yellow oil. ${ }^{1} \mathrm{H} \mathrm{NMR}\left(400 \mathrm{MHz}, \mathrm{CDCl}_{3}\right) \delta 8.31(\mathrm{~s}, 1 \mathrm{H})$, $7.71(\mathrm{~d}, J=7.6 \mathrm{~Hz}, 1 \mathrm{H}), 7.24-7.33(\mathrm{~m}, 2 \mathrm{H}), 7.19-7.21(\mathrm{~m}, 1 \mathrm{H})$, $3.87(\mathrm{~d}, J=5.6 \mathrm{~Hz}, 1 \mathrm{H}), 2.54(\mathrm{~s}, 3 \mathrm{H}), 1.75-1.84(\mathrm{~m}, 1 \mathrm{H}), 0.90(\mathrm{~d}$, $J=6.8 \mathrm{~Hz}, 3 \mathrm{H}), 0.71(\mathrm{~d}, J=6.8 \mathrm{~Hz}, 3 \mathrm{H}) .{ }^{13} \mathrm{C}\{1 \mathrm{H}\} \mathrm{NMR}(100$ $\left.\mathrm{MHz}, \mathrm{CDCl}_{3}\right) \delta 178.8(\mathrm{q}, J=34 \mathrm{~Hz}), 168.6,141.9(\mathrm{q}, J=5 \mathrm{~Hz})$, $135.3,131.0,129.6,127.0,126.5,121.8,119.1,116.9$ (q, $J=290$ $\mathrm{Hz}), 42.7,34.4,24.2,19.9$, 18.4. HRMS (+ESI-TOF) $\mathrm{m} / \mathrm{z}:[\mathrm{M}+\mathrm{H}]^{+}$ Calcd for $\mathrm{C}_{16} \mathrm{H}_{17} \mathrm{~F}_{3} \mathrm{NO}_{2}$ 312.1206; Found 312.1197.

Methyl 4-isopropyl-1-methyl-1,4-dihydroquinoline-3carboxylate (11a): A dry and argon flushed $25 \mathrm{ml}$ sealed tube, equipped with a magnetic stirring bar, was charged with a suspension of $\mathrm{NaH}(37 \mathrm{mg}, 1.52 \mathrm{mmol})$ in DMF $(0.25 \mathrm{ml})$. A solution of compound 3a $(100 \mathrm{mg}, 0.38 \mathrm{mmol})$ in $2.5 \mathrm{ml}$ of wet DMF $(0.1 \%$ v/ $\mathrm{v}_{2} \mathrm{O}$ ) was added dropwise over the $\mathrm{NaH}$ suspension, followed by methyl iodide $(216 \mathrm{mg}, 1.52 \mathrm{mmol})$. The reaction mixture was stirred at $80{ }^{\circ} \mathrm{C}$ for $1 \mathrm{~h}$. After cooled down to $0{ }^{\circ} \mathrm{C}$, excess $\mathrm{NaH}$ was quenched by careful addition of water. The mixture was adjusted to $\mathrm{pH} 7 \sim 8$ by $1 \mathrm{~N} \mathrm{HCl}$, then extracted with EA three times. The combined organic phase was washed with brine, dried over $\mathrm{Na}_{2} \mathrm{SO}_{4}$, and concentrated in vacuo to give the crude product. Purification by flash chromatography (PE:EA $=50: 1 \sim 1: 1$ ) furnishes the desired product ( $80 \mathrm{mg}, 84 \%$ yield) as a yellow oil. ${ }^{1} \mathrm{H}$ NMR $\left(400 \mathrm{MHz}, \mathrm{CDCl}_{3}\right) \delta 7.46(\mathrm{~s}, 1 \mathrm{H}), 7.19-7.23(\mathrm{~m}, 1 \mathrm{H}), 7.09$ $7.10(\mathrm{~m}, 1 \mathrm{H}), 7.02-7.05(\mathrm{~m}, 1 \mathrm{H}), 6.85(\mathrm{~d}, J=8.4 \mathrm{~Hz}, 1 \mathrm{H}), 3.81(\mathrm{~d}$, $J=4 \mathrm{~Hz}, 1 \mathrm{H}), 3.73(\mathrm{~s}, 3 \mathrm{H}), 3.28(\mathrm{~s}, 3 \mathrm{H}), 1.73-1.77(\mathrm{~m}, 1 \mathrm{H}), 0.83$ $(\mathrm{d}, J=7.2 \mathrm{~Hz}, 3 \mathrm{H}), 0.63(\mathrm{~d}, J=6.8 \mathrm{~Hz}, 3 \mathrm{H}) .{ }^{13} \mathrm{C}\{1 \mathrm{H}\} \mathrm{NMR}(100$ $\left.\mathrm{MHz}, \mathrm{CDCl}_{3}\right) \delta 168.2,143.2,139.7 .130 .1,126.7,124.3,122.6$, 111.6, 100.6, 50.8, 42.3, 38.5, 35.7, 19.3, 17.5. HRMS (+ESI-TOF) $m / z:[\mathrm{M}+\mathrm{Na}]^{+}$Calcd for $\mathrm{C}_{15} \mathrm{H}_{19} \mathrm{NO}_{2} \mathrm{Na} 268.1308$; Found 268.1304.

Ethyl 1-ethyl-4-isopropyl-1,4-dihydroquinoline-3-carboxylate (11b): A dry and argon flushed $25 \mathrm{ml}$ sealed tube, equipped with a magnetic stirring bar, was charged with a suspension of $\mathrm{NaH}(90$ $\mathrm{mg}, 3.72 \mathrm{mmol})$ in DMF $(0.6 \mathrm{ml})$. A solution of compound 3a ( $250 \mathrm{mg}, 0.93 \mathrm{mmol})$ in $6.2 \mathrm{ml}$ of wet DMF $\left(0.1 \% \mathrm{v} / \mathrm{v} \mathrm{H}_{2} \mathrm{O}\right)$ was added dropwise over the $\mathrm{NaH}$ suspension, followed by ethyl iodide $(584 \mathrm{mg}, 3.72 \mathrm{mmol})$. The reaction mixture was stirred at $80{ }^{\circ} \mathrm{C}$ for $1 \mathrm{~h}$. After cooled down to $0{ }^{\circ} \mathrm{C}$, excess $\mathrm{NaH}$ was quenched by careful addition of water. The mixture was adjusted to $\mathrm{pH} 7 \sim 8$ by $1 \mathrm{~N} \mathrm{HCl}$, then extracted with EA three times. The combined organic phase was washed with brine, dried over $\mathrm{Na}_{2} \mathrm{SO}_{4}$, and concentrated in vacuo to give the crude product. Purification by flash chromatography (PE:EA $=50: 1 \sim 1: 1$ ) furnishes the desired product $\left(230 \mathrm{mg}, 91 \%\right.$ yield) as a yellow oil. ${ }^{1} \mathrm{H}$ NMR (400 MHz, $\left.\mathrm{CDCl}_{3}\right) \delta 7.48(\mathrm{~s}, 1 \mathrm{H}), 7.16-7.20(\mathrm{~m}, 1 \mathrm{H}), 7.09$ $(\mathrm{d}, J=6.4 \mathrm{~Hz}, 1 \mathrm{H}), 6.99-7.02(\mathrm{~m}, 1 \mathrm{H}), 6.87(\mathrm{~d}, J=8.0 \mathrm{~Hz}, 1 \mathrm{H})$, 4.15-4.25 (m, 2H), 3.75-3.83 (m, 2H), 3.54-3.63 (s, 1H), 1.71-1.79 $(\mathrm{m}, 1 \mathrm{H}), 1.30(\mathrm{t}, J=7.2 \mathrm{~Hz}, 6 \mathrm{H}), 0.82(\mathrm{~d}, J=6.8 \mathrm{~Hz}, 3 \mathrm{H}), 0.64(\mathrm{~d}$, $J=7.2 \mathrm{~Hz}, 3 \mathrm{H}) .{ }^{13} \mathrm{C}\{1 \mathrm{H}\} \mathrm{NMR}\left(100 \mathrm{MHz}, \mathrm{CDCl}_{3}\right) \delta 167.6,141.7$, 138.1, 130.2, 126.5, 124.5, 122.2, 111.6, 100.5, 59.1, 45.2, 42.2, 35.9, 19.1, 17.5, 14.3, 13.1. HRMS (+ESI-TOF) $\mathrm{m} / \mathrm{z}:[\mathrm{M}+\mathrm{Na}]^{+}$ Calcd for $\mathrm{C}_{17} \mathrm{H}_{23} \mathrm{NO}_{2} \mathrm{Na} 296.1621$; Found 296.1605.

2,2,2-Trifluoro-1-(4-isopropyl-1-methyl-1,4-dihydroquinolin-3$y$ l)ethan-1-one (11c): A dry and argon flushed $25 \mathrm{ml}$ sealed tube, equipped with a magnetic stirring bar, was charged with a suspension of $\mathrm{NaH}(108 \mathrm{mg}, 4.48 \mathrm{mmol})$ in DMF $(0.75 \mathrm{ml})$. A solution of compound 3a (300 mg, $1.12 \mathrm{mmol})$ in $7.5 \mathrm{ml}$ of wet DMF $\left(0.1 \% \mathrm{v} / \mathrm{v}_{2} \mathrm{O}\right)$ was added dropwise over the $\mathrm{NaH}$ suspension, and the resulting mixture was stirred at $60{ }^{\circ} \mathrm{C}$ for $30 \mathrm{~min}$. After cooled down to r.t., methyl iodide (190 $\mathrm{mg}, 1.34 \mathrm{mmol})$ was added to the solution and the reaction mixture was stirred at r.t. overnight. The excess $\mathrm{NaH}$ was quenched by careful addition of water. The mixture was adjusted to $\mathrm{pH} 7 \sim 8$ by $1 \mathrm{~N} \mathrm{HCl}$, then extracted with EA three times. The combined organic phase is washed with brine, dried over $\mathrm{Na}_{2} \mathrm{SO}_{4}$, and concentrated in vacuo to give the crude product. Purification by flash chromatography (PE:EA = 50:1 1:1) furnishes the desired product (72 $\mathrm{mg}, 23 \%$ yield) as a yellow solid, as well as the recovered $\mathbf{3 a}(150 \mathrm{mg})$, the yield based on the recovered starting material is $42 \%$. ${ }^{1} \mathrm{H} \mathrm{NMR}(400 \mathrm{MHz}$, 
$\left.\mathrm{CDCl}_{3}\right) \delta 7.56(\mathrm{~s}, 1 \mathrm{H}), 7.25-7.29(\mathrm{~m}, 1 \mathrm{H}), 7.16-7.17(\mathrm{~m}, 2 \mathrm{H}), 6.98$ $(\mathrm{d}, J=8.0 \mathrm{~Hz}, 1 \mathrm{H}), 4.00(\mathrm{~d}, \mathrm{~J}=4.0 \mathrm{~Hz}, 1 \mathrm{H}), 3.44(\mathrm{~s}, 3 \mathrm{H}), 1.71-$ $1.78(\mathrm{~m}, 1 \mathrm{H}), 0.83(\mathrm{~d}, J=6.8 \mathrm{~Hz}, 3 \mathrm{H}), 0.63(\mathrm{~d}, J=6.8 \mathrm{~Hz}, 3 \mathrm{H})$. ${ }^{13} \mathrm{C}\{1 \mathrm{H}\}$ NMR $\left(100 \mathrm{MHz}, \mathrm{CDCl}_{3}\right) \delta 176.2(\mathrm{q}, J=34 \mathrm{~Hz}), 148.5$ $(\mathrm{q}, J=4 \mathrm{~Hz}), 138.1,130.5,127.4,125.2,124.9,117.9$ (q, $J=290$ $\mathrm{Hz}), 112.9,106.5,40.9,39.9,35.4,19.4,17.7$. HRMS (+ESI-TOF) $m / z: \quad[\mathrm{M}+\mathrm{Na}]^{+}$Calcd for $\mathrm{C}_{15} \mathrm{H}_{16} \mathrm{~F}_{3} \mathrm{NONa}$ 306.1076; Found 306.1063 .

1-Benzoyl-4-isopropyl-1,4-dihydroquinoline-3-carboxylic acid (12): To a stirred solution of $9(500 \mathrm{mg}, 1.34 \mathrm{mmol})$ and urea hydrogen peroxide $(524 \mathrm{mg}, 5.56 \mathrm{mmol})$ in DCM $(6.8 \mathrm{ml})$ was added trifluoroacetic anhydride $(1.04 \mathrm{~g}, 4.96 \mathrm{mmol})$ at $0{ }^{\circ} \mathrm{C}$ under argon. The reaction mixture was warmed to r.t. and stirred overnight. The excess peroxide was destroyed by saturated aqueous $\mathrm{Na}_{2} \mathrm{~S}_{2} \mathrm{O}_{3}$ and water. The resulting mixture was extracted with DCM for three times. The combined organic phase was washed with brine, dried over $\mathrm{Na}_{2} \mathrm{SO}_{4}$, filtered and concentrated under reduced pressure to give the crude product. Purification by flash chromatography (PE:EA = 50:1 2:1) furnishes the desired product (283 mg, 66\% yield) as a yellow solid. ${ }^{1} \mathrm{H}$ NMR $(400 \mathrm{MHz}$, $\left.\mathrm{CDCl}_{3}\right) \delta 9.23(\mathrm{~s}, 1 \mathrm{H}), 7.92(\mathrm{~d}, J=7.6 \mathrm{~Hz}, 2 \mathrm{H}), 7.68(\mathrm{~d}, J=7.6$ $\mathrm{Hz}, 1 \mathrm{H}), 7.48-7.51(\mathrm{~m}, 1 \mathrm{H}), 7.39-7.42(\mathrm{~m}, 2 \mathrm{H}), 7.18-7.25(\mathrm{~m}$, $2 \mathrm{H}), 7.11-7.14(\mathrm{~m}, 1 \mathrm{H}), 3.26(\mathrm{~d}, J=10.8 \mathrm{~Hz}, 1 \mathrm{H}), 2.41-2.49(\mathrm{~m}$, $1 \mathrm{H}), 1.00(\mathrm{~d}, J=6.4 \mathrm{~Hz}, 3 \mathrm{H}), 0.59(\mathrm{~d}, J=6.8 \mathrm{~Hz}, 3 \mathrm{H}) .{ }^{13} \mathrm{C}\{1 \mathrm{H}\}$ NMR $\left(100 \mathrm{MHz}, \mathrm{CDCl}_{3}\right) \delta 178.0,166.8,136.0,133.9,132.4$, 130.7, 130.3, 129.9, 129.0, 128.7, 128.5, 127.6, 126.7, 125.9, 57.2, 29.5, 21.9, 20.2. HRMS (-ESI-TOF) $\mathrm{m} / \mathrm{z}$ : [M-H] Calcd for $\mathrm{C}_{20} \mathrm{H}_{18} \mathrm{NO}_{3}$ 320.1292; Found 320.1285.

(4-Isopropyl-3-(1,1,1-trifluoro-2-hydroxy-3-methylbutan-2yl)quinolin-1(4H)-yl)(phenyl)methanone (13): To a solution of compound 9 (100 $\mathrm{mg}, 0.27 \mathrm{mmol})$ in dry THF $(0.5 \mathrm{ml})$ was added isopropylmagnesium chloride lithium chloride solution $(0.24 \mathrm{ml}$, $1.3 \mathrm{M}$ in THF, $0.30 \mathrm{mmol}$ ) dropwise under argon, and the reaction was stirred at r.t. for $2 \mathrm{~h}$. The reaction mixture was quenched by aqueous saturated ammonium chloride solution, diluted with water and extracted with EA three times. The combined organic phase was washed with brine, dried over $\mathrm{Na}_{2} \mathrm{SO}_{4}$, and concentrated in vacuo to give the crude product. Purification by flash chromatography (PE:EA = 100:1 5:1) furnishes the desired product $(73 \mathrm{mg}$, $66 \%$ yield) as a yellow solid. ${ }^{1} \mathrm{H}$ NMR $\left(400 \mathrm{MHz}, \mathrm{CDCl}_{3}\right) \delta 7.62$ (s, 1H), $7.58(\mathrm{~d}, J=7.2 \mathrm{~Hz}, 2 \mathrm{H}), 7.41-7.49(\mathrm{~m}, 3 \mathrm{H}), 7.22(\mathrm{~s}, 1 \mathrm{H})$, 7.09-7.14 (m, 3H), $3.55(\mathrm{~d}, J=3.6 \mathrm{~Hz}, 1 \mathrm{H}), 2.26(\mathrm{~s}, 1 \mathrm{H}), 2.15-$ $2.24(\mathrm{~m}, 1 \mathrm{H}), 1.99-2.06(\mathrm{~m}, 1 \mathrm{H}), 1.06(\mathrm{~d}, J=7.2 \mathrm{~Hz}, 3 \mathrm{H}), 0.93(\mathrm{~d}$, $J=6.4 \mathrm{~Hz}, 3 \mathrm{H}), 0.80(\mathrm{~d}, J=6.8 \mathrm{~Hz}, 3 \mathrm{H}), 0.76(\mathrm{~d}, J=6.8 \mathrm{~Hz}, 3 \mathrm{H})$. ${ }^{13} \mathrm{C}\{1 \mathrm{H}\}$ NMR $\left(100 \mathrm{MHz}, \mathrm{CDCl}_{3}\right) \delta 168.3,138.1,135.9,130.9$, $130.2,129.3,128.5128 .4,126.1,124.9,124.7,122.7,79.4$ (q, $J=$ $27 \mathrm{~Hz}), 46.1,37.2,33.5,21.6,17.3,16.9,16.7$. HRMS (+ESI-TOF) $m / z:[\mathrm{M}+\mathrm{Na}]^{+}$Calcd for $\mathrm{C}_{24} \mathrm{H}_{26} \mathrm{~F}_{3} \mathrm{NO}_{2} \mathrm{Na}$ 440.1808; Found 440.1807 .

(4-Isopropyl-3-(2,2,2-trifluoro-1-hydroxyethyl)quinolin-1(4H)$y l)($ phenyl)methanone (14): To a solution of compound 9 (90 mg, $0.24 \mathrm{mmol})$ in methanol $(1.2 \mathrm{ml})$ was added sodium borohydride (9 $\mathrm{mg}, 0.24 \mathrm{mmol}$ ) slowly, and the reaction mixture was stirred at r. t. for $2 \mathrm{~h}$. The reaction was quenched by $1 \mathrm{~mol} / \mathrm{L} \mathrm{HCl}$, diluted with water and extracted with EA three times. The combined organic phases are washed with brine, dried over $\mathrm{Na}_{2} \mathrm{SO}_{4}$, and concentrated in vacuo to give the crude product. Purification by flash chromatography (PE:EA $=50: 1 \sim 3: 1$ ) furnishes the desired product (75 mg, 83\% yield) as a yellow solid. The product is a mixture of diastereomers (ratio $=1: 0.6)$. Some of the characteristic peaks for the major component: ${ }^{1} \mathrm{H}$ NMR $\left(400 \mathrm{MHz}, \mathrm{CDCl}_{3}\right) \delta 3.17(\mathrm{~d}, J=6$ $\mathrm{Hz}, 1 \mathrm{H}), 2.99(\mathrm{~s}, 1 \mathrm{H}), 1.98-2.06(\mathrm{~m}, 1 \mathrm{H}), 1.08-1.1 .10(\mathrm{~m}, 3 \mathrm{H})$, $0.90(\mathrm{~d}, J=6.4 \mathrm{~Hz}, 3 \mathrm{H}) .{ }^{13} \mathrm{C} \mathrm{NMR}\left(100 \mathrm{MHz}, \mathrm{CDCl}_{3}\right) \delta 71.0(\mathrm{q}, J$ $=32 \mathrm{~Hz}), 48.1,33.9,29.8,21.0,19.4$. Some of the characteristic peaks for the major component: ${ }^{1} \mathrm{H}$ NMR $\left(400 \mathrm{MHz}, \mathrm{CDCl}_{3}\right) \delta$ $3.46(\mathrm{~d}, J=4.8 \mathrm{~Hz}, 1 \mathrm{H}), 2.76(\mathrm{~s}, 1 \mathrm{H}), 2.12-2.17(\mathrm{~m}, 1 \mathrm{H}), 1.08$ - $1.10(\mathrm{~m}, 3 \mathrm{H}), 0.85(\mathrm{~d}, J=6.8 \mathrm{~Hz}, 3 \mathrm{H}) .{ }^{13} \mathrm{C}\{1 \mathrm{H}\} \mathrm{NMR}(100 \mathrm{MHz}$, $\left.\mathrm{CDCl}_{3}\right) \delta 72.1(\mathrm{q}, J=32 \mathrm{~Hz}), 46.4,34.0,30.4,21.5,18.3$. HRMS (+ESI-TOF) $m / z:[\mathrm{M}+\mathrm{Na}]^{+}$Calcd for $\mathrm{C}_{21} \mathrm{H}_{20} \mathrm{NO}_{2} \mathrm{~F}_{3} \mathrm{Na} 398.1338$; Found 398.1326.

1-Acetyl-4-isopropyl-1,4-dihydroquinolin-3(2H)-one (15): To a stirred solution of $10(150 \mathrm{mg}, 0.49 \mathrm{mmol})$ and urea hydrogen peroxide (192 mg, $2.04 \mathrm{mmol})$ in DCM $(2.5 \mathrm{ml})$ was added trifluoroacetic anhydride $(383 \mathrm{mg}, 1.82 \mathrm{mmol})$ at $0^{\circ} \mathrm{C}$ under argon. The reaction mixture was warmed to r.t. and stirred overnight. The excess peroxide was destroyed by saturated aqueous $\mathrm{Na}_{2} \mathrm{~S}_{2} \mathrm{O}_{3}$ and water. The resulting mixture was extracted with DCM for three times. The combined organic phases were washed with brine, dried over $\mathrm{Na}_{2} \mathrm{SO}_{4}$, filtered and concentrated under reduced pressure to give the crude product. Purification by flash chromatography (PE:EA = 50:1 2:1) furnishes the desired product $(34 \mathrm{mg}$, $30 \%$ yield) as a yellow oil. ${ }^{1} \mathrm{H}$ NMR $\left(400 \mathrm{MHz}, \mathrm{CDCl}_{3}\right) \delta 7.36-$ $7.38(\mathrm{~m}, 1 \mathrm{H}), 7.23-7.29(\mathrm{~m}, 2 \mathrm{H}), 7.15-7.17(\mathrm{~m}, 1 \mathrm{H}), 5.30(\mathrm{~d}, J=$ $19.2 \mathrm{~Hz}, 1 \mathrm{H}), 3.57(\mathrm{~d}, J=18.8 \mathrm{~Hz}, 1 \mathrm{H}), 3.07(\mathrm{~d}, J=9.6 \mathrm{~Hz}, 1 \mathrm{H})$, $3.23(\mathrm{~s}, 3 \mathrm{H}), 1.93-1.99(\mathrm{~m}, 1 \mathrm{H}), 1.03(\mathrm{~d}, J=6.4 \mathrm{~Hz}, 3 \mathrm{H}), 0.83(\mathrm{~d}$, $J=6.8 \mathrm{~Hz}, 3 \mathrm{H}) .{ }^{13} \mathrm{C}\{1 \mathrm{H}\} \mathrm{NMR}\left(100 \mathrm{MHz}, \mathrm{CDCl}_{3}\right) \delta 207.7,169.6$, 138.1, 132.0, 130.8, 128.2, 127.2, 125.5, 63.4, 51.1, 30.5, 22.1, 21.4, 20.9. HRMS (+ESI-TOF) $\mathrm{m} / \mathrm{z}: \quad[\mathrm{M}+\mathrm{Na}]^{+}$Calcd for $\mathrm{C}_{14} \mathrm{H}_{17} \mathrm{NO}_{2} \mathrm{Na} 254.1152$; Found 254.1143.

Methyl 5-cyano-1-methyl-4-(o-tolyl)-1,4-dihydropyridine-3carboxylate (16): A dry and argon flushed $25 \mathrm{ml}$ sealed tube, equipped with a magnetic stirring bar, was charged with a suspension of $\mathrm{NaH}(83 \mathrm{mg}, 3.44 \mathrm{mmol})$ in DMF $(0.58 \mathrm{ml})$. A solution of compound 3p (250 mg, $0.86 \mathrm{mmol})$ in $5.8 \mathrm{ml}$ of wet DMF $(0.1 \%$ $\mathrm{v} / \mathrm{v}_{2} \mathrm{O}$ ) was added dropwise over the $\mathrm{NaH}$ suspension, followed by methyl iodide ( $489 \mathrm{mg}, 3.44 \mathrm{mmol}$ ). The reaction mixture was stirred at $80{ }^{\circ} \mathrm{C}$ for $4 \mathrm{~h}$. After cooled down to $0{ }^{\circ} \mathrm{C}$, excess $\mathrm{NaH}$ was quenched by careful addition of water. The mixture was adjusted to $\mathrm{pH} 7 \sim 8$ by $1 \mathrm{~N} \mathrm{HCl}$, then extracted with EA three times. The combined organic phase was washed with brine, dried over $\mathrm{Na}_{2} \mathrm{SO}_{4}$, and concentrated in vacuo to give the crude product. Purification by flash chromatography (PE:EA $=50: 1 \sim 2: 1$ ) furnishes the desired product $(210 \mathrm{mg}, 91 \%$ yield) as a yellow solid. ${ }^{1} \mathrm{H}$ NMR $\left(400 \mathrm{MHz}, \mathrm{CDCl}_{3}\right) \delta$ 7.15-7.22 (m, 3H), 7.01-7.13 (m, $2 \mathrm{H}), 6.51(\mathrm{~d}, J=1.6 \mathrm{~Hz}, 1 \mathrm{H}), 4.89(\mathrm{~s}, 1 \mathrm{H}), 3.58(\mathrm{~s}, 3 \mathrm{H}), 3.21(\mathrm{~s}$, $3 \mathrm{H}), 2.54(\mathrm{~s}, 3 \mathrm{H}) .{ }^{13} \mathrm{C}\{1 \mathrm{H}\} \mathrm{NMR}\left(100 \mathrm{MHz}, \mathrm{CDCl}_{3}\right) \delta 166.7$, $144.0,139.6,138.4,134.9,130.1,129.1,127.0,126.7,119.4$, 106.7, 90.2, 51.4, 41.4, 34.0, 19.4. HRMS (+ESI-TOF) $\mathrm{m} / \mathrm{z}$ : $[\mathrm{M}+\mathrm{Na}]^{+}$Calcd for $\mathrm{C}_{16} \mathrm{H}_{16} \mathrm{~N}_{2} \mathrm{O}_{2} \mathrm{Na} 291.1104$; Found 291.1089.

4-(O-tolyl)-5-(2,2,2-trifluoro-1-hydroxyethyl)-1,4-

dihydropyridine-3-carbonitrile (17): To a solution of compound $3 \mathbf{p}(100 \mathrm{mg}, 0.35 \mathrm{mmol})$ in methanol $(1.7 \mathrm{ml})$ was added sodium borohydride (14 mg, $0.35 \mathrm{mmol})$ slowly, and the reaction mixture was stirred at r. t. overnight. The reaction was quenched by 1 $\mathrm{mol} / \mathrm{L} \mathrm{HCl}$, diluted with water and extracted with EA three times. The combined organic phases are washed with brine, dried over $\mathrm{Na}_{2} \mathrm{SO}_{4}$, and concentrated in vacuo to give the crude product. Purification by flash chromatography (PE:EA $=50: 1 \sim 2: 1$ ) furnishes the desired product ( $83 \mathrm{mg}, 82 \%$ yield) as a yellow oil. The product is a mixture of diastereomers (ratio $\approx 1: 1)$. ${ }^{1} \mathrm{H}$ NMR (400 $\left.\mathrm{MHz}, \mathrm{CDCl}_{3}\right) \delta 7.45(\mathrm{~d}, J=7.6 \mathrm{~Hz}, 1 \mathrm{H}), 7.38(\mathrm{~d}, J=7.6 \mathrm{~Hz}, 1 \mathrm{H})$, $7.20-7.26(\mathrm{~m}, 2 \mathrm{H}), 7.17(\mathrm{~d}, J=4 \mathrm{~Hz}, 4 \mathrm{H}), 6.69(\mathrm{t}, J=4.8 \mathrm{~Hz}, 2 \mathrm{H})$, $6.58(\mathrm{~d}, J=4.8 \mathrm{~Hz}, 1 \mathrm{H}), 6.42(\mathrm{~d}, J=4.8 \mathrm{~Hz}, 1 \mathrm{H}), 5.92(\mathrm{~s}, 1 \mathrm{H})$, $5.85(\mathrm{~s}, 1 \mathrm{H}), 4.81(\mathrm{~s}, 1 \mathrm{H}), 4.70(\mathrm{~s}, 1 \mathrm{H}), 4.20-4.25(\mathrm{~m}, 1 \mathrm{H}), 4.03-$ $4.05(\mathrm{~m}, 1 \mathrm{H}), 2.48(\mathrm{~s}, 3 \mathrm{H}), 2.42(\mathrm{~s}, 3 \mathrm{H}), 2.20(\mathrm{~d}, J=3.6 \mathrm{~Hz}, 1 \mathrm{H})$, $1.74(\mathrm{~d}, J=7.6 \mathrm{~Hz}, 1 \mathrm{H}) .{ }^{13} \mathrm{C}\{1 \mathrm{H}\}$ NMR $\left(100 \mathrm{MHz}, \mathrm{CDCl}_{3}\right) \delta$ $142.0,137.4,137.0,135.6,131.5,131.0,130.8,130.3,129.9$, $129.5,127.8,127.7,127.5,127.3,127.2,126.0,125.8,125.7$, $124.7,123.1,123.0,120.5,120.3,109.5,108.7,85.5,84.4,72.4$ (q, $J=32 \mathrm{~Hz}), 68.8(\mathrm{q}, J=32 \mathrm{~Hz}), 19.3$. HRMS (+ESI-TOF) $\mathrm{m} / \mathrm{z}$ : $[\mathrm{M}+\mathrm{Na}]^{+}$Calcd for $\mathrm{C}_{15} \mathrm{H}_{13} \mathrm{~N}_{2} \mathrm{OF}_{3} \mathrm{Na}$ 317.0872; Found 317.0860. 
1-Acetyl-4-(o-tolyl)-5-(2,2,2-trifluoroacetyl)-1,4-

dihydropyridine-3-carbonitrile (18): To a solution of $\mathbf{3 p}(2 \mathrm{~g}, 6.85$ mmol) in dry DCM (14 ml) was added acetyl chloride $(1.6 \mathrm{~g}$, $20.55 \mathrm{mmol}$ ) dropwise, followed by DMAP (166 mg, $1.37 \mathrm{mmol})$ and triethylamine (2.1 g, $20.55 \mathrm{mmol})$. The reaction mixture was stirred at r.t. overnight. Then it is quenched with water and extracted with EA three times. The combined organic phase was washed with brine, dried over $\mathrm{Na}_{2} \mathrm{SO}_{4}$, and concentrated in vacuo to give the crude product. Purification by flash chromatography (PE:EA = 50:1 1:1) furnishes the desired product $(1.4 \mathrm{~g}, 62 \%$ yield) as a yellow solid. ${ }^{1} \mathrm{H}$ NMR (400 MHz, d6-DMSO) $\delta 8.33(\mathrm{~s}$, $1 \mathrm{H}), 8.01(\mathrm{~s}, 1 \mathrm{H}), 7.16-7.25(\mathrm{~m}, 4 \mathrm{H}), 4.96(\mathrm{~s}, 1 \mathrm{H}), 2.54(\mathrm{~s}, 3 \mathrm{H})$, $2.52(\mathrm{~s}, 3 \mathrm{H}) .{ }^{13} \mathrm{C}\{1 \mathrm{H}\} \mathrm{NMR}(100 \mathrm{MHz}, \mathrm{d} 6-\mathrm{DMSO}) \delta 177.8(\mathrm{q}, J$ $=33 \mathrm{~Hz}), 168.3,140.7,135.4,135.2,133.7,130.1,128.6,127.6$, 127.0, 117.5, 116.1 (q, $J=290 \mathrm{~Hz}), 114.4,96.3,34.0,21.7,19.1$. HRMS (+ESI-TOF) m/z: $[\mathrm{M}+\mathrm{K}]^{+}$Calcd for $\mathrm{C}_{17} \mathrm{H}_{13} \mathrm{~N}_{2} \mathrm{O}_{2} \mathrm{~F}_{3} \mathrm{~K}$ 373.0561; Found 373.0566.

(O-tolyl)-5-(1,1,1-trifluoro-2-hydroxypropan-2-yl)-1,4dihydropyridine-3-carbonitrile (19): To a solution of compound $18(100 \mathrm{mg}, 0.30 \mathrm{mmol})$ in dry THF $(1.5 \mathrm{ml})$ was added methyl lithium solution $(0.75 \mathrm{ml}, 1.3 \mathrm{M}$ in ethyl ether, $0.99 \mathrm{mmol})$ dropwise under argon, and the reaction was stirred at r.t. for $1 \mathrm{~h}$. The reaction mixture was quenched by aqueous saturated ammonium chloride solution, diluted with water and extracted with EA three times. The combined organic phase was washed with brine, dried over $\mathrm{Na}_{2} \mathrm{SO}_{4}$, and concentrated in vacuo to give the crude product. Purification by flash chromatography (PE:EA $=30: 1 \sim 1: 1$ ) furnishes the desired product (70 $\mathrm{mg}, 76 \%$ yield) as a yellow solid. ${ }^{1} \mathrm{H}$ NMR $\left(400 \mathrm{MHz}, \mathrm{CD}_{3} \mathrm{OD}\right) \delta 7.35(\mathrm{~d}, J=7.6 \mathrm{~Hz}, 1 \mathrm{H}), 7.16-$ $7.20(\mathrm{~m}, 1 \mathrm{H}), 7.07-7.13(\mathrm{~m}, 2 \mathrm{H}), 6.85(\mathrm{~d}, J=5.2 \mathrm{~Hz}, 2 \mathrm{H}), 4.58(\mathrm{~s}$, $1 \mathrm{H}), 2.45(\mathrm{~s}, 3 \mathrm{H}), 0.98(\mathrm{~s}, 3 \mathrm{H}) .{ }^{13} \mathrm{C}\{1 \mathrm{H}\} \mathrm{NMR}\left(100 \mathrm{MHz}, \mathrm{CD}_{3} \mathrm{OD}\right)$ $\delta 146.7,139.4,131.4,130.3,128.0,127.9,127.52(\mathrm{q}, J=284 \mathrm{~Hz})$, 127.51, 122.4, 113.5, 84.2, 74.7 (q, $J=28 \mathrm{~Hz}), 23.0,19.5$. HRMS (+ESI-TOF) $m / z$ : $[\mathrm{M}+\mathrm{Na}]^{+}$Calcd for $\mathrm{C}_{16} \mathrm{H}_{15} \mathrm{~N}_{2} \mathrm{OF}_{3} \mathrm{Na} 331.1029$; Found 331.1016.

\section{ASSOCIATED CONTENT}

\section{Supporting Information}

The Supporting Information is available free of charge on the ACS Publications website.

Experimental procedures and characterization data for all new compounds (PDF).

\section{AUTHOR INFORMATION}

\section{Corresponding Author \\ * E-mail: wangdong@tust.edu.cn}

\section{ORCID}

Dong Wang: 0000-0002-4855-0576

\section{Notes}

The authors declare no competing financial interests.

\section{ACKNOWLEDGMENT}

We thank the National Key R\&D Program of China (2018YFA0901700) and the National Natural Science Foundation of China (81673296) for generous financial support. We thank the reviewers for helpful suggestions.

\section{REFERENCES}

(1) Campos, K. R.; Coleman, P. J.; Alvarez, J. C.; Dreher, S. D.; Garbaccio, R. M.; Terrett, N. K.; Tillyer, R. D.; Truppo, M. D.; Parmee, E. R. The importance of synthetic chemistry in the pharmaceutical industry. Science 2019, 363, eaat0805.

(2) (a) Bossert, F.; Vater, W. 1,4-Dihydropyridines--a basis for developing new drugs. Med Res Rev 1989, 9, 291-324; (b) Janis, R. a.; Silver, P. J.; Triggle, D. J. Drug action and cellular calcium regulation. Adv. Drug Res. 1987, 16, 309-591.

(3) (a) Knaus, T.; Paul, C. E.; Levy, C. W.; de Vries, S.; Mutti, F. G.; Hollmann, F.; Scrutton, N. S. Better than Nature: Nicotinamide Biomimetics That Outperform Natural Coenzymes. J. Am. Chem. Soc. 2016, 138, 1033-1039; (b) Paul, C. E.; Gargiulo, S.; Opperman, D. J. Lavandera, I.; Gotor-Fernandez, V.; Gotor, V.; Taglieber, A.; Arends, I. W. C. E.; Hollmann, F. Mimicking Nature: Synthetic Nicotinamide Cofactors for $\mathrm{C}=\mathrm{C}$ Bioreduction Using Enoate Reductases. Org. Lett. 2013, 15, 180-183.

(4) Meyers, A. I.; Oppenlaender, T. Efficient chirality transfer between a chiral 4-methyl-1,4-dihydropyridine and benzoylformic ester. An example of a pure intermolecular self-immolative process. $J$. Am. Chem. Soc. 1986, 108, 1989-1996.

(5) (a) Hadida, S.; Van Goor, F.; Zhou, J.; Arumugam, V.; McCartney, J.; Hazlewood, A.; Decker, C.; Negulescu, P.; Grootenhuis, P. D. J. Discovery of N-(2,4-Di-tert-butyl-5-hydroxyphenyl)-4-oxo-1,4dihydroquinoline-3-carboxamide (VX-770, Ivacaftor), a Potent and Orally Bioavailable CFTR Potentiator. J. Med. Chem. 2014, 57, 97769795; (b) Mistry, S. N.; Valant, C.; Sexton, P. M.; Capuano, B.; Christopoulos, A.; Scammells, P. J. Synthesis and Pharmacological Profiling of Analogues of Benzyl Quinolone Carboxylic Acid (BQCA) as Allosteric Modulators of the M1 Muscarinic Receptor. J. Med. Chem. 2013, 56, 5151-5172; (c) Zhang, R.; Han, G.; Jiang, L.; Shen, Y.; Yang, R. Mao, Y.; Wang, H. An Efficient Synthesis of Ivacaftor. J. Heterocycl. Chem. 2017, 54, 3169-3173; (d) El-Essawy, F. A.; Boshta, N. M.; Alotaibi, M. A.; Elsayed, M. S.; Tarabees, R.; Saleh, E. A. Construction of 1,3-disubstituted 4-oxo-1,4-dihydroquinolines as a potential antibacterial agents. Res. Chem. Intermed. 2016, 42, 8157-8174.

(6) (a) Carelli, V.; Liberatore, F.; Scipione, L.; Di Rienzo, B.; Tortorella, S. Dithionite adducts of pyridinium salts: regioselectivity of formation and mechanisms of decomposition. Tetrahedron 2005, 61, 10331-10337; (b) Wong, Y. S.; Marazano, C.; Gnecco, D.; Das, B. C. 1,4Dihydropyridines from dithionite reduction of pyridinium salts without electron-withdrawing groups as substituents. Tetrahedron Lett. 1994, 35, 707-710.

(7) (a) Charette, A. B.; Grenon, M.; Lemire, A.; Pourashraf, M.; Martel, J. Practical and Highly Regio- and Stereoselective Synthesis of 2-Substituted Dihydropyridines and Piperidines: Application to the Synthesis of (-)-Coniine. J. Am. Chem. Soc. 2001, 123, 11829-11830; (b) Fernandez-Ibanez, M. A.; Macia, B.; Pizzuti, M. G.; Minnaard, A. J.; Feringa, B. L. Catalytic Enantioselective Addition of Dialkylzinc Reagents to N-Acylpyridinium Salts. Angew. Chem., Int. Ed. 2009, 48, 9339-9341; (c) Donohoe, T. J.; Connolly, M. J.; Walton, L. Regioselective Nucleophilic Addition to Pyridinium Salts: A New Route to Substituted Dihydropyridones. Org. Lett. 2009, 11, 5562-5565; (d) Christian, N.; Aly, S.; Belyk, K. Rhodium-Catalyzed Enantioselective Addition of Boronic Acids to N-Benzylnicotinate Salts. J. Am. Chem. Soc. 2011, 133, 2878-2880; (e) Chau, S. T.; Lutz, J. P.; Wu, K.; Doyle, A. G. Nickelcatalyzed enantioselective arylation of pyridinium ions: harnessing an iminium ion activation mode. Angew. Chem., Int. Ed. 2013, 52, 91539156; (f) Lutz, J. P.; Chau, S. T.; Doyle, A. G. Nickel-catalyzed enantioselective arylation of pyridine. Chem. Sci. 2016, 7, 4105-4109; (g) Bull, J. A.; Mousseau, J. J.; Pelletier, G.; Charette, A. B. Synthesis of pyridine and dihydropyridine derivatives by regio- and stereoselective addition to N-activated pyridines. Chem. Rev. 2012 $112,2642-2713$.

(8) Grozavu, A.; Hepburn, H. B.; Smith, P. J.; Potukuchi, H. K.; Lindsay-Scott, P. J.; Donohoe, T. J. The reductive C3 functionalization of pyridinium and quinolinium salts through iridium-catalysed interrupted transfer hydrogenation. Nat. Chem. 2019, 11, 242-247.

(9) (a) Bennasar, M. L.; Vidal, B.; Bosch, J. Nucleophilic Addition of 2-Acetylindole Enolates to Pyridinium Salts. Acylation of the Intermediate Dihydropyridines. J. Org. Chem. 1995, 60, 4280-4286; 
(b) Bennasar, M. L.; Juan, C.; Bosch, J. Synthesis of 3,5-diacyl-4-phenyl1,4-dihydropyridines. Tetrahedron Lett. 1998, 39, 9275-9278.

(10) Gribble, M. W.; Guo, S.; Buchwald, S. L. Asymmetric CuCatalyzed 1,4-Dearomatization of Pyridines and Pyridazines without Preactivation of the Heterocycle or Nucleophile. J. Am. Chem. Soc. 2018, 140, 5057-5060.

(11) (a) Wang, S.-G.; You, S.-L. Hydrogenative Dearomatization of Pyridine and Asymmetric Aza-Friedel-Crafts Alkylation Sequence. Angew. Chem., Int. Ed. 2014, 53, 2194-2197; (b) Wang, S.-G.; Zhang, W.; You, S.-L. Construction of Spiro-tetrahydroquinolines via Intramolecular Dearomatization of Quinolines: Free of a Preinstalled Activation Group. Org. Lett. 2013, 15, 1488-1491; (c) Yang, Z.-P.; Wu, Q.-F.; You, S.-L. Direct asymmetric dearomatization of pyridines and pyrazines by iridium-catalyzed allylic amination reactions. Angew. Chem., Int. Ed. 2014, 53, 6986-6989; (d) Yang, Z.-P.; Wu, Q.-F.; Shao, W.; You, S.-L. Iridium-catalyzed intramolecular asymmetric allylic dearomatization reaction of pyridines, pyrazines, quinolines, and isoquinolines. J. Am. Chem. Soc. 2015, 137, 15899-15906.

(12) (a) Wang, D.; Liu, Z.; Wang, Z.; Ma, X.; Yu, P. Metal- and basefree regioselective thiolation of the methyl $\mathrm{C}(\mathrm{sp} 3)-\mathrm{H}$ bond in 2 picoline N-oxides. Green Chem. 2019, 21, 157-163; (b) Wang, D. Wang, Z.; Liu, Z.; Huang, M.; Hu, J.; Yu, P. Strategic C-C Bond-Forming Dearomatization of Pyridines and Quinolines. Org. Lett. 2019, 21 4459-4463; (c) Wang, D.; Ma, X.; Dong, L.; Feng, H.; Yu, P.; Désaubry, L. Stereoselective Four-Component Synthesis of Functionalized 2,3Dihydro-4-Nitropyrroles. Frontiers in Chemistry 2019, 7:810; (d) Wang, D.; Wang, Y.; Zhao, J.; Shen, M.; Hu, J.; Liu, Z.; Li, L.; Xue, F.; Yu, P. Strategic Approach to 8-Azacoumarins. Org. Lett. 2017, 19, 984-987; (e) Wang, D.; Feng, H.; Li, L.; Liu, Z.; Yan, Z.; Yu, P. Access to 8Azachromones via Activation of $\mathrm{C}-\mathrm{H}$ in N-Oxides. J. Org. Chem. 2017, $82,11275-11287$.

(13) (a) Kelly, C. B.; Mercadante, M. A.; Leadbeater, N. E. Trifluoromethyl ketones. Properties, preparation, and application. Chem. Commun. 2013, 49, 11133-11148; (b) Schlosser, M. CF3-bearing aromatic and heterocyclic building blocks. Angew. Chem., Int. Ed. 2006, 45, 5432-5446.

(14) Chen, Q.; Mollat du Jourdin, X.; Knochel, P. Transition-metalfree BF3-mediated regioselective direct alkylation and arylation of functionalized pyridines using Grignard or organozinc reagents. J. Am. Chem. Soc. 2013, 135, 4958-4961.

(15) For selective examples, see: (a) Chipanina, N. N.; Oznobikhina, L. P.; Aksamentova, T. N.; Romanov, A. R.; Rulev, A. Y. Intramolecular hydrogen bond in the push-pull CF3-aminoenones: DFT and FTIR study, NBO analysis. Tetrahedron 2014, 70, 1207-1213; (b) Brewster, M. E.; Kaminski, J. J.; Huang, M. J.; Bodor, N. Reactivity of biologically important reduced pyridines. 7. Energetics and effect of substitution on hydride versus electron transfer in dihydropyridines, dihydroquinolines, and dihydroisoquinolines. J. Org. Chem. 1990, 55, 2361-6.

(16) Delgado, A.; Clardy, J. Aryl trifluoromethyl ketone hydrates as precursors of carboxylic acids and esters. Tetrahedron Lett. 1992, 33, $2789-2790$.

(17) (a) Amoah, E.; Dieter, R. K. Regioselective 1,4-Conjugate Addition of Grignard Reagents to $\alpha, \beta-\gamma, \delta$-Dienones and $\alpha, \beta-\gamma, \delta$-Dienyl Thiol Esters. J. Org. Chem. 2017, 82, 2870-2888; (b) Holm, T. Mechanism of the Grignard addition reaction. XVI. Homolytic and concerted mechanisms in the reaction of $\alpha, \beta$-unsaturated carbonyl compounds with Grignard reagents. Acta Chem. Scand. 1991, 45, 925929.

(18) Mellor, J. M.; Reid, G.; El-Sagheer, A. H.; El-Tamany, E.-S. H. Reaction of Alkyl and Aryl Grignard Reagents with Trifluoroacetyldihydropyrans and Other Cyclic $\beta$-Alkoxy- $\alpha, \beta-$ unsaturated Trifluoromethylketones. Tetrahedron 2000, 56, 1003910055.

(19) Romanov, A. R.; Cahard, D.; Rulev, A. Y. Regioselectivity Issues in the Addition of Grignard Reagents to Trifluoromethylated $\alpha$ Bromoenones. Eur. J. Org. Chem. 2019, 2019, 2143-2149.

(20) Okano, T.; Matsubara, H.; Kusukawa, T.; Fujita, M. The polar effect on the regiochemistry of nucleophilic substitution of trifluoromethylated $\pi$-allylpalladium complex. J. Organomet. Chem. 2003, 676, 43-48. 